\title{
PASSIVE CABLE PROPERTIES OF DENDRITIC SPINES AND SPINY NEURONS ${ }^{1}$
}

\author{
CHARLES J. WILSON ${ }^{2}$
}

Department of Anatomy, Michigan State University, East Lansing, Michigan 48824

Received April 6, 1983; Revised August 25, 1983; Accepted August 26, 1983

\begin{abstract}
A cable model of the linear properties of dendritic spines was generated using the Laplace transform technique. Analytical solutions for the voltages generated in the spine by a current impulse at the spine head were used in a numerical procedure for simulating the effect of a synaptic conductance change. The synaptic current produced by the conductance change was used as an input for evaluation of the postsynaptic potential and current injected into the dendrite at the base of the spine.

The primary effect of the dendritic spine was to attenuate synaptic current. This effect was produced by the high input impedance at axospinous synapses, which resulted in giant spike-like excitatory postsynaptic potentials (EPSPs) that approached the reversal potential of the synapse and thus reduced the potential gradient driving the synaptic current. Although virtually all of the synaptic current was transferred to the dendrite, it produced much smaller EPSPs there due to the low dendritic input impedance. Very small conductance changes produced near maximal synaptic currents in dendritic spines. The current attenuating effect of the spine was accentuated with brief synaptic transients and reduced with prolonged synaptic conductance changes. The size and shape of the spine head, and the diameter and boundary conditions of the dendrite had little or no effect on current attenuation for spines in the naturally occurring size range. The diameter and length of the spine stalk and the size and location of the spine apparatus were the key morphological factors determining the synaptic currents generated by axospinous synapses. Naturally occurring size and shape differences among dendritic spines produced large differences in synaptic potency when compared in a model spiny neuron based on the neostriatal spiny projection neuron. These differences were comparable to those produced by differences in synaptic location on the same neuron.
\end{abstract}

Each neuron's unique set of integrative properties is at least partly determined by its size and shape. Although the shapes of neurons are probably a factor in a wide variety of their cellular functions, the most well known effects are on spatial summation of synaptic inputs delivered to different regions of the cell. A number of theoretical predictions of the relative attenuation of synaptic potentials generated at different locations on well studied neurons have proven accurate in experimental tests (e.g., Rall et al., 1967; Iansek and Redman, 1973;

\footnotetext{
${ }^{1}$ I thank Dr. P. M. Groves and S. T. Kitai for the use of their computing facilities at various times during the development of the programs, and Drs. R. J. MacGregor and S. J. Young for their help in getting started. A preliminary report was presented at the meeting of the Society for Neuroscience, November 1982. Supported by National Institutes of Health Grant NS 17294.

${ }^{2}$ Present address: Department of Anatomy, University of Tennessee Center for Health Science, Memphis, TN 38163.
}

Barrett and Crill, 1974; Tsukahara et al., 1975). Because of these successes, the notion of weighting of inputs by synaptic location has become a generally accepted principle of neuronal organization. An attractive possibility suggested by this principle is that even the relatively subtle specializations of surface morphology traditionally used to characterize neuron types may directly reflect the special functional properties of the neurons possessing them.

While there are more elaborate neuronal surface specializations, the most common are short thin appendages arising from dendrites or somata and receiving only one or a few synaptic contacts. On many neurons in mammalian forebrain and on Purkinje cells of cerebellar cortex these are called dendritic spines, and they have a very stereotyped structure and distribution on the dendrites. More variably shaped but similar postsynaptic specializations found throughout the brainstem and spinal cord may be located on somata or dendrites (for 
review see Scheibel and Scheibel, 1968). In all cases examined in detail the spines have proven to be specialized zones of synaptic contact, and synapses are often scant or absent along the intervening neuronal membrane (e.g., Gray, 1959).

Since the suggestion was made by Chang (1952) that dendritic spines might attenuate synaptic potentials, numerous authors have speculated on the relative effectiveness of axospinous and axodendritic synaptic contacts. More recently, a number of more detailed theoretical discussions of dendritic spines have emphasized various aspects of the idea that the high longitudinal resistance of dendritic spines might affect synaptic transmission (Llinás and Hillman, 1969; Diamond et al., 1970; Rall, 1974, 1978; Jack et al., 1975; Shepherd and Brayton, 1979; Rinzel, 1982; Koch and Poggio, 1983). At the same time evidence from many sources has indicated that dendritic spines may change shape and size in response to changing physiological conditions or pathological states (e.g., Globus and Scheibel, 1967; Valverde, 1967; Purpura, 1974; Van Harreveld and Fifková, 1975; Coss and Globus, 1978). Most of the theoretical simulations have employed constant rather than time varying inputs, however, and in all the results suggested that synaptic effects on dendritic spines may depend critically upon several geometrical and clectrical characteristics. The interpretation of the empirical data on spine shape changes thus requires that new attention be paid to two problems. It is necessary to obtain the most accurate possible measurements of the dimensions of dendritic spines and the electrical properties of the spine membranes. It is also desirable for theoretical simulations to be applied to specific cases as carefully and accurately as possible so that testable quantitative predictions can be made. Because of the difficulty of obtaining estimates of many of the variable quantities, however, it is important to identify the most critical parameters and those that may have little effect on the predicted outcome.

A general examination of all of the relevant passive parameters was the aim of the work reported here. The method is exactly that described by Jack et al. (1975), but it has been extended to include transient synaptic conductances. The range of morphological variables was derived from measurements of dendritic spines of rat neostriatal spiny neurons (Wilson et al., 1983). Membrane electrical parameters were based on measurements from these same cells but were extended to cover a range suitable to a large variety of spiny mammalian neurons.

\section{Materials and Methods}

Assumptions and notation. The dendritic spine was treated as consisting of two spatially distributed compartments as shown in Figure 1. The spine head was considered to be isopotential and so could be treated as a simple parallel RC network with its diameter as its single geometrical parameter. The spine stalk and each of the two dendritic segments were assumed to be uniform cylinders of passive linear membrane. The extracellular fluid was treated as isopotential. Equalization of membrane potential around the circumference of the cylindrical compartments was assumed to be rapid compared to longitudinal spread, allowing application of onedimensional cable theory (Rall, 1969). These assumptions are the ones generally employed in models of passive membrane properties, and they have been discussed at length by others (e.g., Jack et al., 1975; Rall, 1977). In addition to these, however, the dendrites were treated as identical and infinite in length. This led to considerable simplification of the solutions to the characteristic responses and was considered acceptable because the properties of the spine rather than the dendrite were in question. The problem of dendritic termination was considered separately as described under "Results."

Characteristic responses. Analytical solutions to the voltage response across the membrane of the spine head and at the junction of spine stalk and dendrites (spine base) and the current response at the base of the spine upon occurrence of a current impulse (Dirac delta function) were obtained using the Laplace transform method. The solutions are closely related to those of Jack and
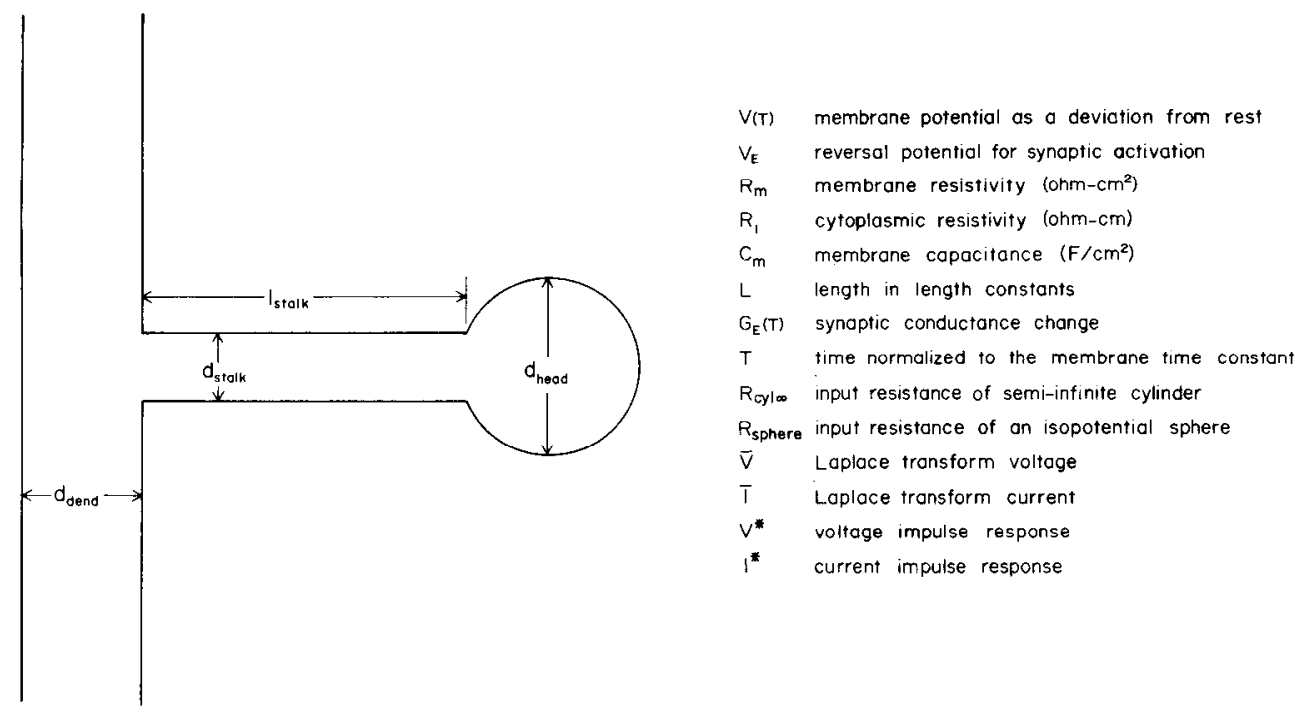

Figure 1. Notation 
Redman (1971) and were obtained in the same way, as shown under "Appendix."

They are:

$$
\begin{aligned}
V_{\text {head }}^{*}= & \frac{R_{\text {head }} e^{-T}}{(\pi / 2)^{1 / 2}} \\
& \cdot\left[\sum_{n=0}^{\infty} b^{n} e^{-X 1^{2}} \sum_{r=0}^{n} \frac{(-n)_{r}}{r !}(2 a)^{r}(2 T)^{r / 2}\right. \\
& \cdot e^{\left(W 1^{2 / 4}\right]} D_{-(r+1)}[W 1]-\sum_{n=0}^{\infty} b^{n+1} e^{-X 2^{2}} \sum_{r=0}^{n} \frac{(-n)_{r}}{r !} \\
V_{\text {base }}^{*}= & \frac{R_{\text {head }}(1-b) e^{-T}}{(\pi / 2)^{1 / 2}} \sum_{n=0}^{\infty} b^{n} e^{-X 3^{2}} \\
& \cdot \sum_{r=0}^{n} \frac{(-n)_{r}}{r !}(2 a)^{r}(2 T)^{r / 2} e^{\left[W 3^{2} / 4\right]} D_{-(r+1)}[W 3] \\
I_{\text {base }}^{*}= & \frac{a(1+b)^{-r}}{(\pi T)^{1 / 2}} \\
& \cdot\left[\sum_{n=0}^{\infty} b^{n} e^{-X 3^{2}} \sum_{r=0}^{n}(r+1) \frac{(-n)_{r}}{r !}(2 a)^{r}(2 T)^{r / 2}\right. \\
& \cdot e^{\left[W 3^{2} / 4\right]} D_{-(r+2)}[W 3]+\sum_{n=0}^{\infty} b^{n} e^{-X 3^{2}} \sum_{r=0}^{n}(X 3) \frac{(-n)_{r}}{r !} \\
& \left.\cdot(2 a)^{r}(2 T)^{r / 2} e^{\left(W 3^{2} / 4\right]} D_{-(r+1)}[W 3]\right], \\
&
\end{aligned}
$$

where

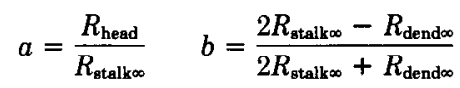

and

$$
\begin{array}{lll}
X 1=\frac{2 n L_{\text {stalk }}}{(2 T)^{1 / 2}} & X 2=\frac{2(n+1) L_{\text {stalk }}}{(2 T)^{1 / 2}} & X 3=\frac{(2 n+1) L_{\text {stalk }}}{(2 T)^{1 / 2}} \\
W 1=(2 T)^{1 / 2} a+X 1 & W 2=(2 T)^{1 / 2} a+X 2 & W 3=(2 T)^{1 / 2} a+X 3
\end{array}
$$

The parameter $a$ is analogous to $\rho_{\infty}$ of Jack and Redman (1971) and $\rho$ of Rall (1959) and represents the stalk to head conductance ratio if the stalk were infinitely long. The other parameter $b$ is related to the branching ratio of Rall and Rinzel (1973) and defines the relationship between the diameters of the stalk and the two dendritic segments. When the dendrites become vanishingly small, $b=-1$ and the solutions reduce to those obtained by Jack and Redman (1971) for an isopotential soma (represented by the spine head) attached to a single short dendrite (the spine stalk) with an insulated termination. If the dendritic input conductance becomes infinitely large, $b=1$ and the solutions reduce to the Jack and Redman (1971) model for the same arrangement but a shorted dendritic termination. If $b=0$ the dendrites can be treated as an equivalent cylinder of the same diameter as the spine stalk. The spine and dendrite are then electrotonically continuous, and the solutions are those of an isopotential sphere attached to a semi-infinite cable dendrite (Rall, 1960; Jack and Redman 1971). A similar result is obtained if stalk length becomes vanishingly short (see "Appendix") or at values of time sufficiently long to allow equalization of charge over the spine membrane (see below).

If the ratio a becomes large enough (the spine head conductance becomes vanishingly small), the solutions reduce to a much simpler form.

$$
\begin{aligned}
V_{\text {head }}^{*} & =\frac{R_{\text {stalk.o }} e^{-T}}{(\pi T)^{1 / 2}} \sum_{n=0}^{\infty}(-b)^{n} e^{-X 1^{2}}-(-b)^{n+1} e^{-X 2^{2}} \\
V_{\text {base }}^{*} & =\frac{R_{\text {stalk } \infty}(1-b) e^{-T}}{(\pi T)^{1 / 2}} \sum_{n=0}^{\infty}(-b)^{n} e^{-X 3^{2}} \\
I_{\text {hese }}^{*} & =\frac{(1+b) e^{-T}}{(2 T)^{1 / 2}(\pi T)^{1 / 2}} \sum_{n=0}^{\infty}(-b)^{n}(X 3) e^{-X 3^{2}}
\end{aligned}
$$

These simplified forms of the solutions are presented because they were found to be adequate to describe nearly all of the predicted properties of spines in the naturally occurring range of spine head diameters (see "Results").

Finally, when sufficient time has passed to allow equalization of charge over the entire spine membrane,

$$
\begin{gathered}
V_{\text {head }}^{*}=V_{\text {base }}^{*}=\frac{R_{\text {bead }} e^{-T}}{1+a L_{\text {stalk }}} e^{\left[Y^{2} T\right]} \operatorname{erfc}[Y \sqrt{T}] \\
I_{\text {bame }}^{*}=\frac{Y e^{-T}}{a}\left[\frac{1}{(\pi T)^{1 / 2}}-Y e^{\left[Y^{2} T\right]} \operatorname{erfc}[Y \sqrt{T}]\right]
\end{gathered}
$$

where

$$
\begin{aligned}
& Y=\frac{(1+b) a}{(1-b)\left(1+a L_{\text {stelk }}\right)}, \\
& \text { and erfc is the complementary error function. }
\end{aligned}
$$

These solutions are of practical importance because all of the previous series converge more slowly with larger values of time, and they become especially difficult when the spine becomes isopotential. Because of the small surface area of the spine, this generally occurs rapidly in comparison to charge redistribution times on the larger dendritic surfaces. To save computation time and avoid rounding errors in the long series approximations, convergence to these asymptotes was monitored and they were employed to complete characteristic responses to the required values of $T(0.2$ to 1.0$)$. Figure 2 is an example showing the head and base voltage responses of a long thin spine for which equalization of charge occurs relatively slowly. The asymptotic curve is also shown. The usefulness of the asymptotic solution is evident from the convergence of the base solution and the nearly complete convergence of the head solution to the asymptote by 0.02 membrane time constants.

Synaptic conductances and synaptic currents. Excitatory synaptic activation was simulated by a time varying conductance added to that of the head membrane. Through this synaptic conductance could flow currents proportional to the conductance and to the difference between the membrane potential and a synaptic reversal potential. This latter was always $70 \mathrm{mV}$ positive to the resting potential. Synaptic currents and postsynaptic potentials at the spine head were evaluated in a stepwise fashion from the characteristic voltage response at the spine head and the synaptic conductance. The synaptic current at each time point was calculated using the membrane potential already calculated for that point. 
This current was then used to obtain the value of the membrane potential at the next time point and so on, as indicated below.

$$
\begin{aligned}
I_{j} & =\text { synaptic current at } T=(j+0.5) \Delta T \\
V_{j} & =\text { membrane potential at } T=(j+0.5) \Delta T \\
V_{j}^{*} & =\text { characteristic voltage response at input at } T=(j+0.5) \Delta T \\
G E_{j} & =\text { synaptic conductance at } T=(j+0.5) \Delta T \\
V_{o} & =0 \\
I_{j} & =G E_{j}\left(V E-V_{j}\right) \\
V_{j+1} & =\sum_{i=0}^{j} I_{j-i} V_{i}^{*} \Delta T
\end{aligned}
$$

The time varying synaptic current produced in this way was used to generate driven base voltage and base current responses from the appropriate characteristic responses by numerical convolution. Synaptic conductances were generated using the so-called alpha function (e.g., Jack and Redman, 1971) and are shown in Figure 3.

Computational methods. Characteristic responses for head voltage, base voltage, and base current were evaluated on a CDC 6400 or a PDP-11/03 computer using double precision arithmetic. Parabolic cylinder functions were generated by the backward recursion method described by Abramowitz and Stegun (1970, pp. 697-700). Convergence of infinite series solutions was evaluated using the criterion that the last term must make a less than $0.1 \%$ change in the series approximation. Convergence to the asymptotic solutions was evaluated using a

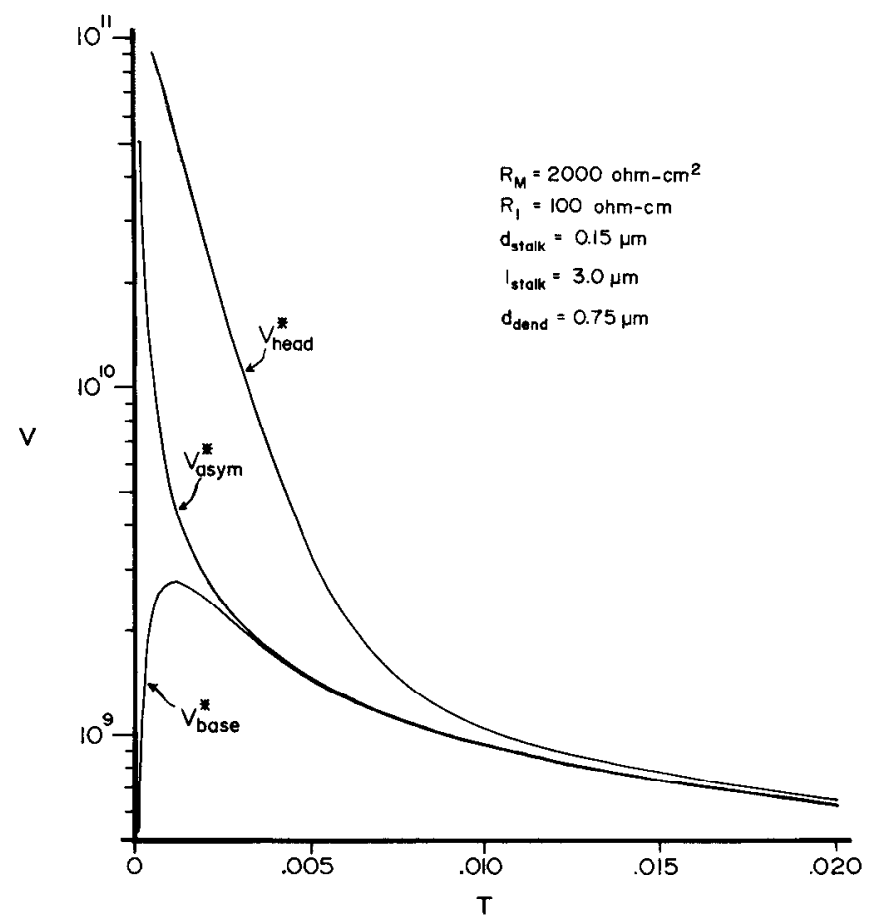

Figure 2. Characteristic (impulse) responses of membrane potential at the spine head and base in a representative spine over the time course of charge redistribution on the spine membrane. The asymptotic response, approached as the spine becomes isopotential, is also shown.
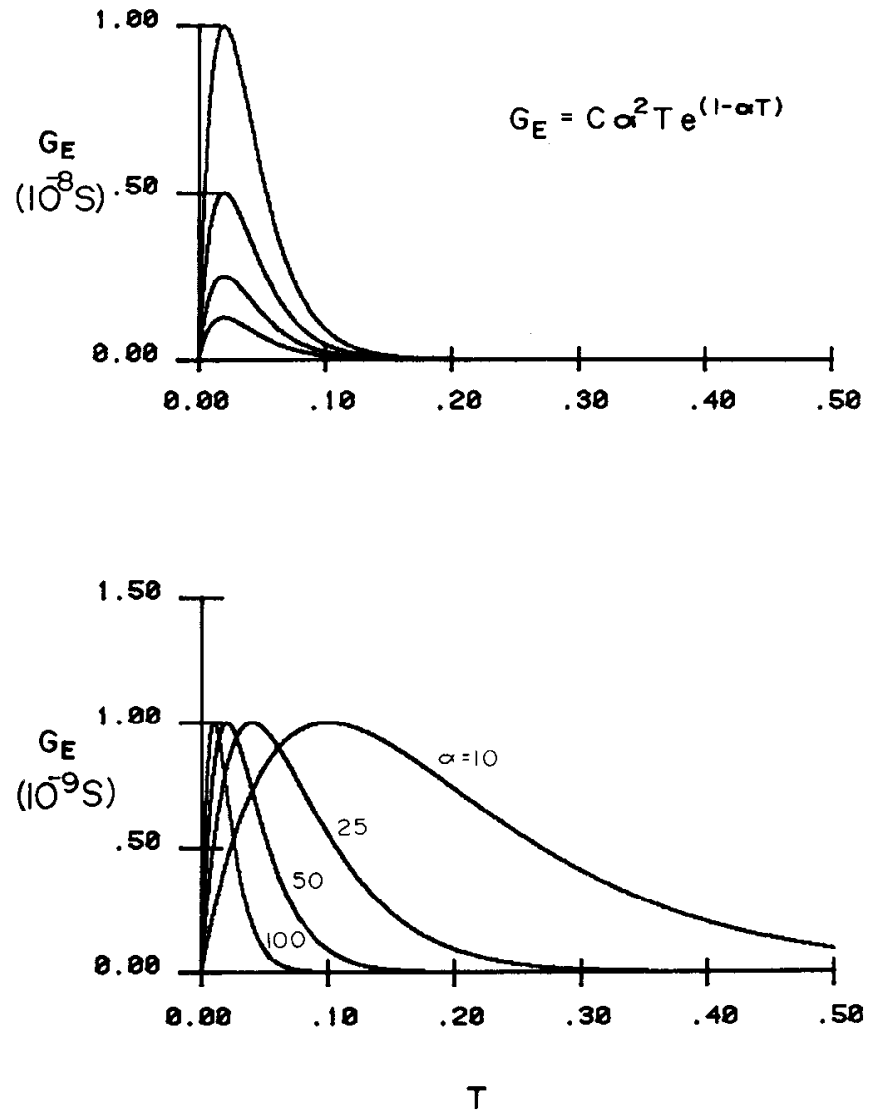

Figure 3. Curves used to represent the time course of the synaptic conductance change and their generating function. Peak conductance (upper graph) and conductance time course (lower graph) were varied independently. Peak conductances occur at time $1 / \alpha$ and have the value of $\mathrm{C} \alpha$.

$1.0 \%$ criterion. The function $\exp \left[z^{2}\right] \operatorname{erfc}[z]$ was evaluated using two series for numerical approximation (Abramowitz and Stegun, 1970, pp. 297-298).

$$
\begin{aligned}
& e^{\left(z^{2}\right]} \operatorname{erfc}[z]=e^{\left[z^{2}\right]}-\frac{2}{(\pi)^{1 / 2}} \sum_{n=0}^{\infty} \frac{2^{n}[z]^{2 n+1}}{1 \cdot 3 \cdot 5 \cdot \ldots \cdot(2 n+1)} \quad z \leq 3.0 \\
& e^{\left[z^{2}\right]} \operatorname{erfc}[z]=\frac{1}{z(\pi)^{1 / 2}}\left[1+\sum_{n=1}^{\infty}(-1)^{n} \frac{1 \cdot 3 \cdot 5 \cdot \ldots \cdot(2 n-1)}{\left[2 z^{2}\right]^{n}}\right] \quad z>3.0
\end{aligned}
$$

All computations except those involving the Rinzel and Rall (1974) model were performed using

$$
\begin{aligned}
T_{j} & =(j+0.5) \Delta T \quad j=1,2,3, \ldots 2000 \\
\Delta T & =1.0 \times 10^{-4}
\end{aligned}
$$

The Rinzel and Rall model calculations used

$$
\begin{aligned}
T_{j} & =(j+0.5) \Delta T \quad j=1,2,3, \ldots 2000 \\
\Delta T & =5.0 \times 10^{-4}
\end{aligned}
$$

In the latter case the first one or two time intervals in the characteristic responses were corrected using more accurate estimates obtained by averaging the responses calculated with shorter time intervals. This was necessary because of the importance of the fast components of the characteristic responses, especially at the spine head. 


\section{Results}

Waveform of the synaptic conductance. An example showing the effect of a transient synaptic conductance change at the head of a dendritic spine in the naturally occurring size range is shown in Figure 4. In this example a conductance reaching its peak amplitude of $5 \mathrm{nS}$ at 0.02 time constants after its onset $(\alpha=50)$ was placed on the spine head membrane. Despite its small amplitude, this conductance change produced a local EPSP with a peak amplitude of almost $25 \mathrm{mV}$ and a time course almost identical to the conductance change. This spikelike EPSP owed both its large amplitude and rapid time course to the high input resistance and rapid redistribution of charge that are characteristic of dendritic spines. The dendritic spine thus should exhibit a very high input impedance even to the high frequency components of the input waveform which would otherwise be shunted by the capacitance of the dendrite. The dendrite is unaffected by this, of course, and these components of the EPSP are lost from the synaptic potential as it occurs at the spine base. The dendritic input impedance is much lower than that of the spine at all frequencies, and the base EPSP shown in Figure 4 is much lower in amplitude than that at the spine head. The small area of the dendritic spine membrane does not allow a significant loss of charge through its resistance. In the case shown in Figure 4 , the current flowing across the synaptic conductance was within $1 \%$ of that flowing into the dendrite at all times except very early time points when both currents were very small (not shown). The difference between spine head and base synaptic potentials resulted strictly from the difference in their input impedances. If the same synaptic current generated by the axospinous synapse were placed directly into the dendrite, it would produce the same postsynaptic potential as that produced at the spine base. Thus, if synapses were simulated using a predetermined synaptic current as input, the dendritic spine would appear to have no effect on the EPSP as it would occur in the dendrite or at the soma of the spiny neuron.

The large amplitude of the spine head EPSP shown in Figure 4 suggests that axospinous synapses may exhibit severe non-ohmic behavior that would modify synaptic current, however. As the membrane potential at the spine head approaches the synaptic reversal potential, it will reduce the potential gradient responsible for the synaptic current. Increases in synaptic conductance under these conditions will produce less than proportional increases in synaptic currents. If this nonlinearity occurs on the spine but less or not at all on the dendrite, the spine will act to attenuate synaptic currents and synaptic potentials. Simulated dendritic spines over a wide range of sizes and shapes and electrical parameters exhibited severely nonlinear behavior with increases in synaptic conductance. An example is shown in Figure $5(A$ and $B)$. To make these figures, synaptic currents were generated with and without the nonlinearity produced by the synaptic potential (to remove the nonlinearity, $V_{j}=$ 0 for all $j$ in the current equation, equation 9 under "Materials and Methods"). This is equivalent to comparing a synaptic conductance change to a current injection as input to the dendritic spine. The current attenuating effect of the high input impedance of the axospinous synapse is evident in Figure $5 A$ over the entire range of conductances examined (peak amplitudes from 0.1 to $10.0 \mathrm{nS}$ ). Head and base EPSP amplitudes reflected this decrease in synaptic current (Fig. 5B). The linear (dashed) relationship for base current is approximately the result that would be obtained for an axodendritic synapse over the same range of synaptic conductances. This is because base current is nearly identical to synaptic current and because the dendritic input impedance to these fast transient inputs is relatively low. The EPSP

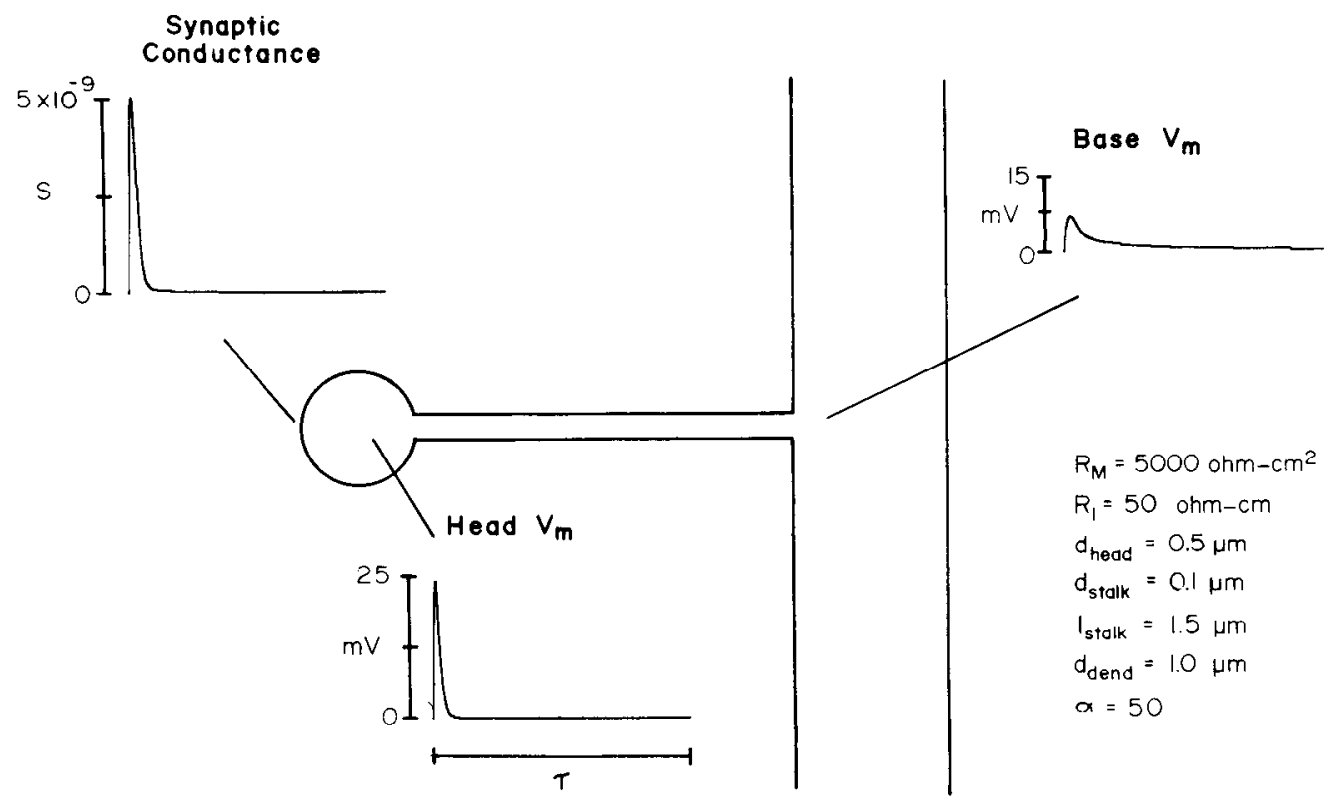

Figure 4. Synaptic potentials generated by a representative simulated axospinous synaptic conductance change. 


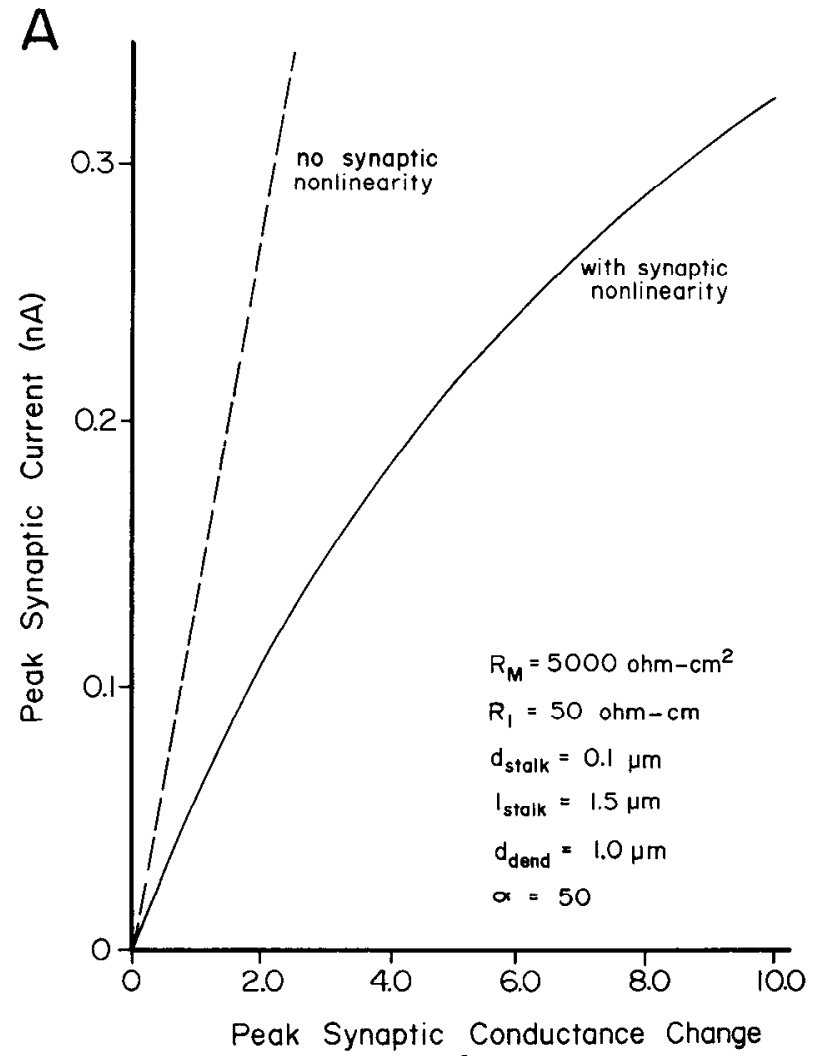
$\left(10^{-9} \mathrm{~S}\right)$

C

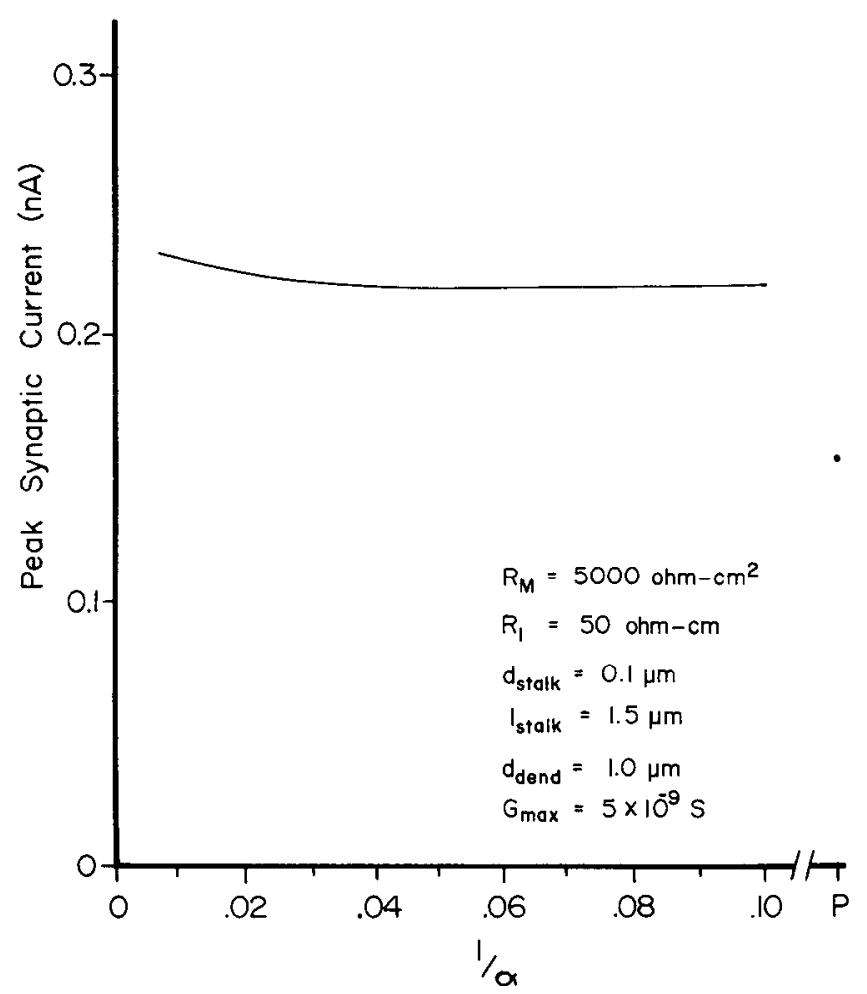

B

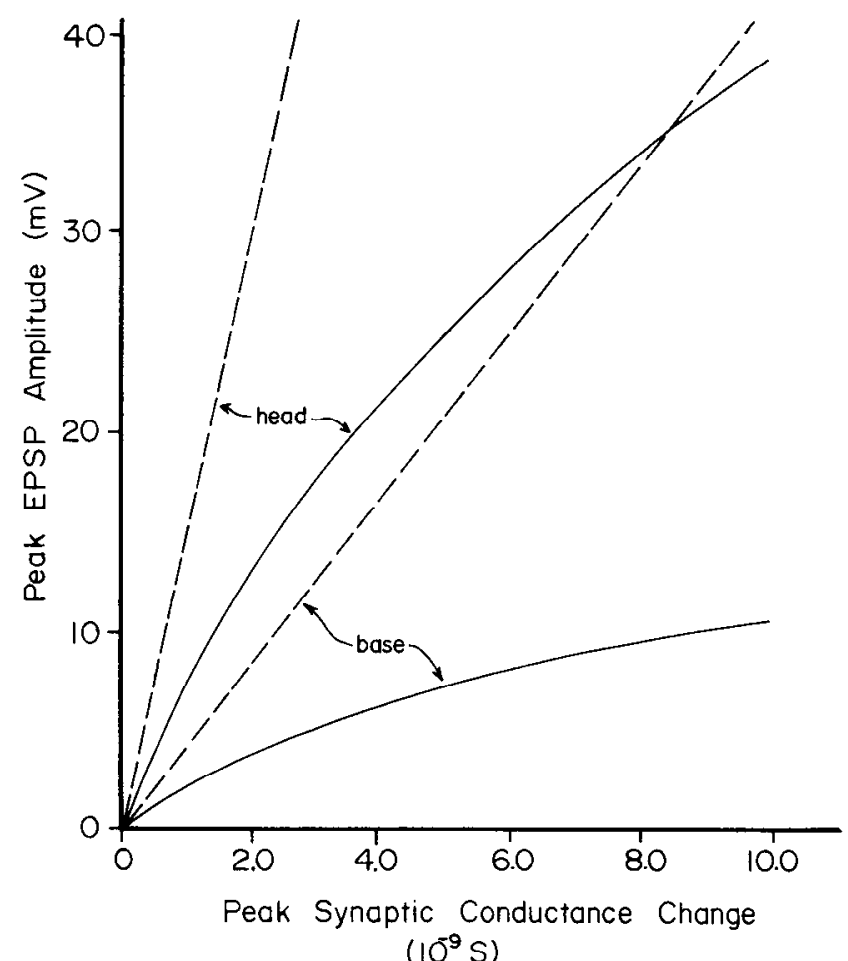

$\left(10^{-9} \mathrm{~S}\right)$

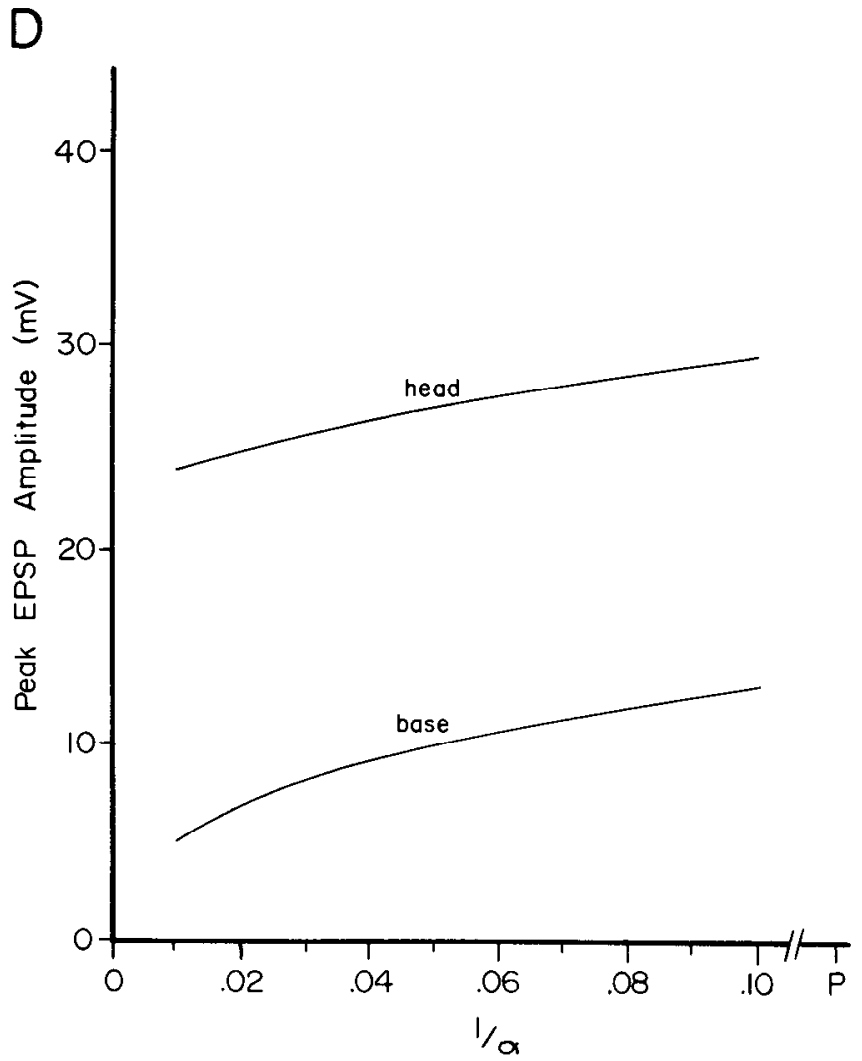

Figure 5. Variation of synaptic current and postsynaptic potentials with peak conductance $(A$ and $B)$ and conductance duration $(C$ and $D)$. Effects in the absence of synaptic nonlinearity are shown as dashed lines. The current attenuating effect of dendritic spines is represented by the deviation from the linear response in $A$. The reduced current produces a saturation-like effect on the postsynaptic potentials $(B)$, even on the relatively small EPSP at the spine base. Conductance durations shown in $C$ and $D$ are the ones used in Figure 3. The abcissa gives time to peak conductance in units of the time constant. The point at $P$ is the steady state response to a steady conductance of the same amplitude as the transients. 
attenuating effect of the dendritic spine, shown as the deviation of the base EPSP from the dashed curve, is, like the synaptic current attenuation that causes it, primarily the result of nonlinearity inherent in the generation of synaptic potentials by conductance changes. It is thus increased disproportionately by increases in synaptic conductance, as can be seen in Figure $5 B$.

It should be noted that the synaptic conductances used here are very small by most standards. For comparison, the conductance change resulting from a single quantal event at the frog's neuromuscular junction is about 100 $\mathrm{nS}$, and that of a single open channel is between 0.01 and $0.1 \mathrm{nS}$ (Takeuchi, 1977).

The effects of changes in the duration of the synaptic conductance are illustrated in Figure $5, C$ and $D$. Over the entire range of synaptic durations, increases in duration of the conductance change increased EPSP amplitudes in both spine head and base and decreased peak synaptic currents. The increased EPSP amplitudes in these cases resulted from the increased input impedance of both spine and dendrite at the lower frequencies contained in the longer duration synaptic transients. For very short durations ( $\alpha$ less than about 50), this tendency was exaggerated due to the reactance of the spine membrane, which becomes significant with very short inputs. This was accompanied by a deviation of the curves for spine head and base EPSPs from their normal parallel trajectory (Fig. $5 D$ )). Over the rest of the range of transients studied (up to $\alpha=10$ ), head and base EPSPs increased gradually with synaptic transient duration and peak current was affected litte. The maximal reduction in peak synaptic current and the maximal peak EPSP amplitudes achieved with an indefinitely prolonged conductance are also shown. The relatively small change in peak synaptic current observed over the examined range of transient synaptic conductances is an indication of an insenitivity of the current attenuating effect of the dendritic spine to small changes in the input duration. Over this range, the conductance change is relatively slow compared to the time course of charge redistribution on the spine, and it is rapid compared to the time course of dendritic charge redistribution. In this range of synaptic conductance change durations, the dendritic spine is most effective at producing finely graded changes in synaptic potency with changes in shape, as described below.

It should be recognized that the ability of axospinous synaptic currents to evoke potential changes in the dendrite at a distance from the spine (for example, at the soma) is critically dependent upon the duration of the current, as well as its amplitude. Small prolonged synaptic currents may produce much greater somatic PSPs than large but very brief ones, depending upon the electronic properties of the dendrites. This is just as true for axodendritic synapses, however, and does not reflect any attribute of the spine. In comparisons of synaptic effectiveness presented below, conductance durations will be kept constant unless otherwise noted, to allow use of peak current as an index of synaptic potency.

Spine head diameter. Increases in the surface area of the isopotential spine head (with no change in the peak synaptic conductance) should be expected to decrease the input resistance of that compartment, resulting in an increase in synaptic current. This did occur, as indicated by the dashed lines in Figure 6. Increases in head diameter past about $0.5 \mu \mathrm{m}$ generally led to increases in synaptic current, especially with the longest (highest resistance) spine stalks. A much smaller, barely noticeable (in Fig. 6) increase in current was obtained with a simple varicosity, or spine head attached directly to the dendrite (stalk length $=0.0$ ). At the same time, however, the increase in membrane surface area offered greater opportunity for leakage of current across the spine head membrane. This effect was also most pronounced in spines with the longest stalks, because of the slower removal of charge from the spine membrane by redistribution onto the dendrite. These two effects of large head diameter were thus directly opposed, and the resulting current injected into the dendrite was almost unaffected by changes in head diameter over the range from zero to $1.5 \mu \mathrm{m}$ (Fig. 6, solid line).

Synaptic currents and spine base currents deviated substantially from each other only in the cases of spines with head diameters exceeding $0.5 \mu \mathrm{m}$. Even in those cases base currents were very close to those obtained with small spine heads. In the data presented in all subsequent figures spine head diameter was set at 0.35 $\mu \mathrm{m}$ or less and will not usually be noted. Synaptic current in those figures is interchangable with base current.

Stalk length and diameter. The dimensions of the spine stalk were the most critical variables determining the attenuation of synaptic currents by dendritic spines. An example showing the effects of stalk diameter and stalk length over the ranges appropriate for neostriatal neu-

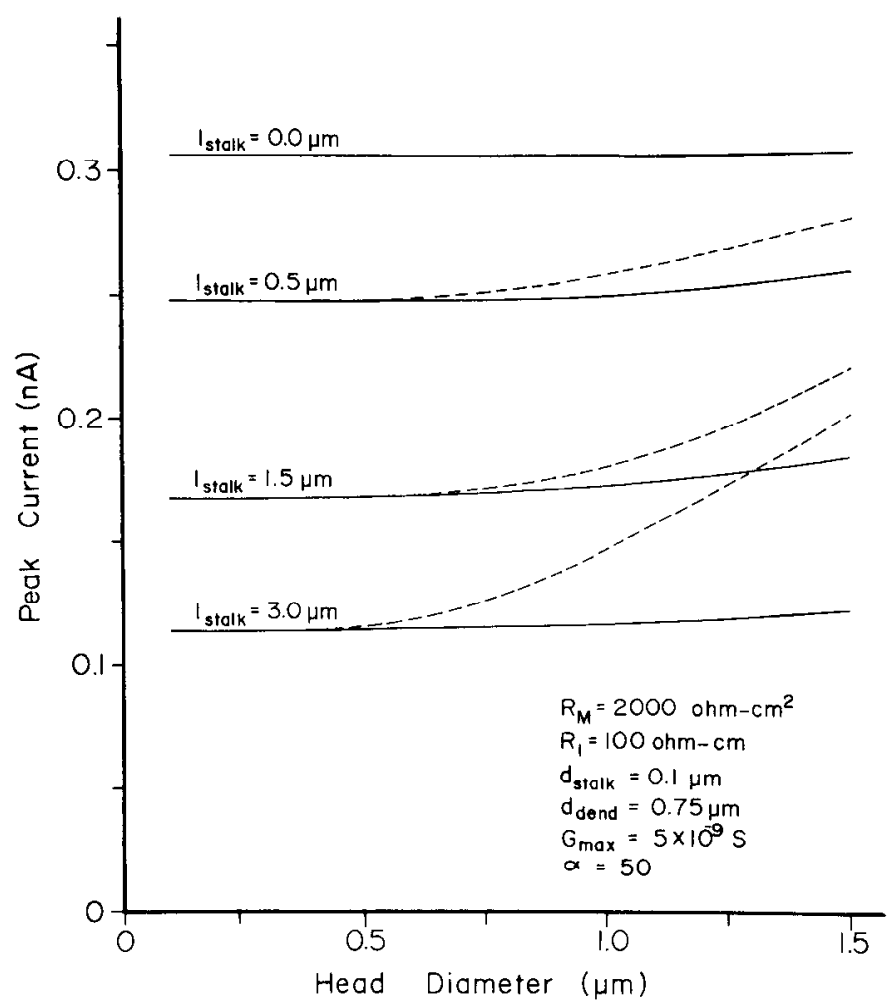

Figure 6. The effect of spine head diameter on peak synaptic current (dashed lines) and current injected into the dendrite (solid lines). Four spine stalk lengths are shown. Note the relatively small effect of the spine head on spine base current. 
rons is shown in Figure 7A. Spines of all lengths shared approximately the same maximal synaptic current achieved with very large stalk diameter. This maximal current was primarily determined by the conductance change parameters and the characteristics of the dendrite. It is very close to the current that would occur if the synaptic conductance were located directly on the dendrite. For long stalks this maximal current was achieved at much larger values of stalk diameter than required for short spines. With extremely fine stalk diameters outside the naturally occurring range, the synaptic currents for spines of all lengths approached zero and each other.

The key role of stalk dimensions was the most robust aspect of the model, being reproduced over a wide range of peak synaptic conductances, membrane and cytoplasmic resistivities (see below), and dendrite and spine head dimensions. It was also relatively persistent in the face of changes in synaptic conductance durations over the range of those shown in Figure 3. With very prolonged synaptic conductances, however, the large difference in the rate of charge redistribution in the spine and dendrite becomes unimportant. As a result, the dendritic input resistance becomes an important factor, and relative importance of the spine stalk is diminished. This is shown in Figure $7 R$ as a decrease in the maximal peak synaptic current and concurrent loss of the range of spine stalk diameters which can effectively attenuate synaptic current. Thus, small differences in spine stalk dimensions within the naturally occurring range will produce large differences in synaptic transmission only with transient synaptic conductances. More prolonged and especially steady conductances will be much less affected by spine geometry.

The reduction in amplitude of the peak synaptic current with prolonged synaptic conductance is of the same type as that shown in Figure $5 \mathrm{C}$ and does not necessarily represent a less effective synaptic current from the point of view of the soma.

Intracellular resistivity and the spine apparatus. An especially difficult parameter to estimate from experimental data is the resistance of the intracellular compartment. Even when the average cytoplasmic resistivity can be measured directly, it cannot be assumed that this will be an accurate estimate for another region of the same cell or even for a small portion of the measured volume where organelles of a particular kind may bc aggregated or cytoskeletal components may be specially organized. Direct measurements of axoplasmic resistivity in invertebrate giant axons reviewed by Rall (1977) vary from 30 to $200 \mathrm{ohm}-\mathrm{cm}$. The effect of cytoplasmic resistivity on current attenuation in dendritic spines was studied over this range. An example is shown in Figure $8 A$. In that figure peak current is plotted against stalk diameter as in Figure 7. Comparison with Figure $7 \mathrm{~A}$ shows that decreasing the intracellular resistivity had approximately the same effect as decreasing the spine length. This similarity was especially pronounced with very long or very thin spines, for which the resistance of the stalk far outweighs any change in the dendritic input
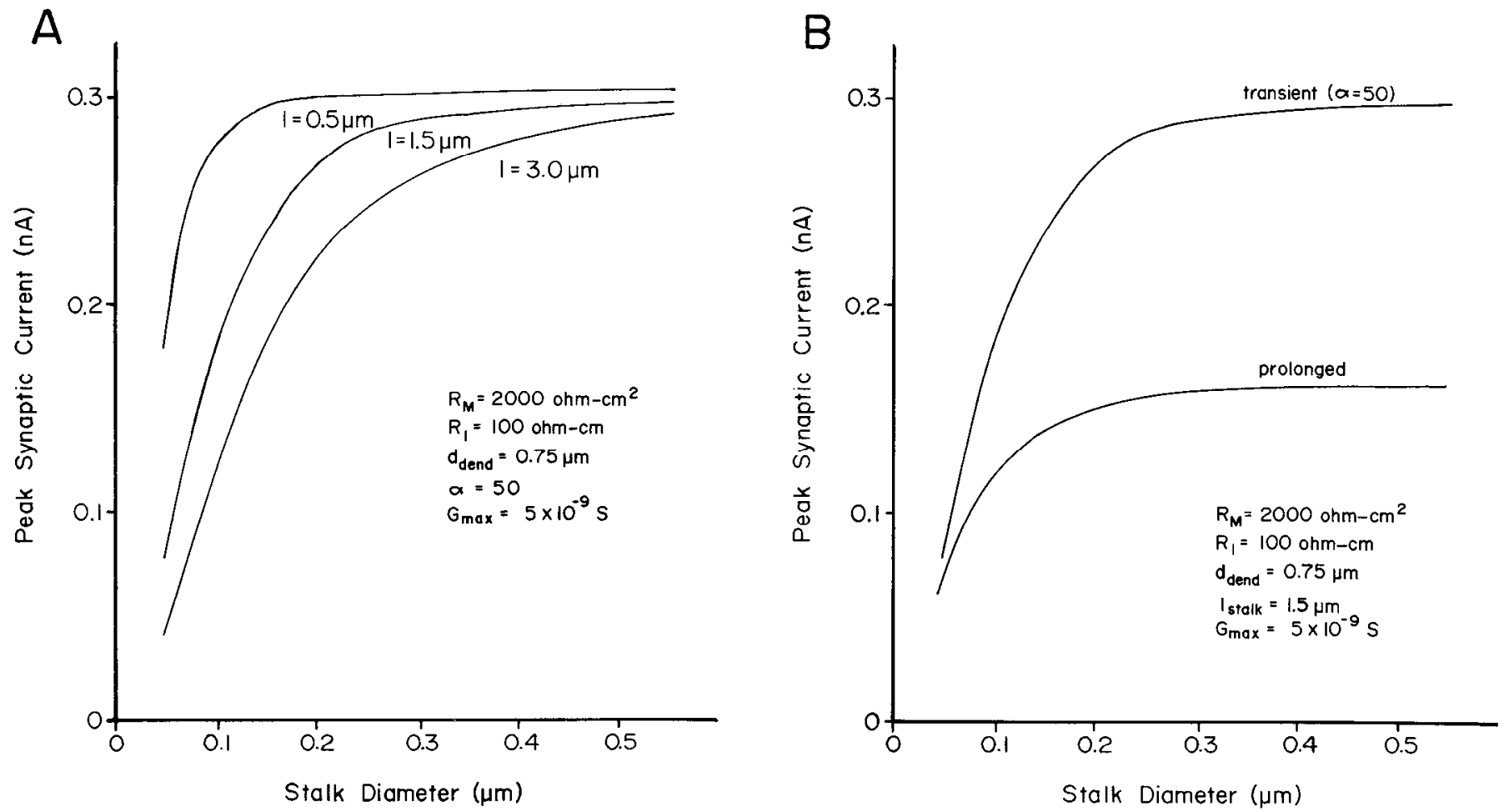

Figure 7. The effect of variation of spine stalk dimensions in the naturally occurring range on peak synaptic current. $A$, Stalk diameter effect for three stalk lengths. Changes in stalk length or diameter produce large effects on synaptic transmission with a transient conductance change. $B$, The same changes are much less pronounced and occur over a smaller range of values with an indefinitely prolonged conductance change. 

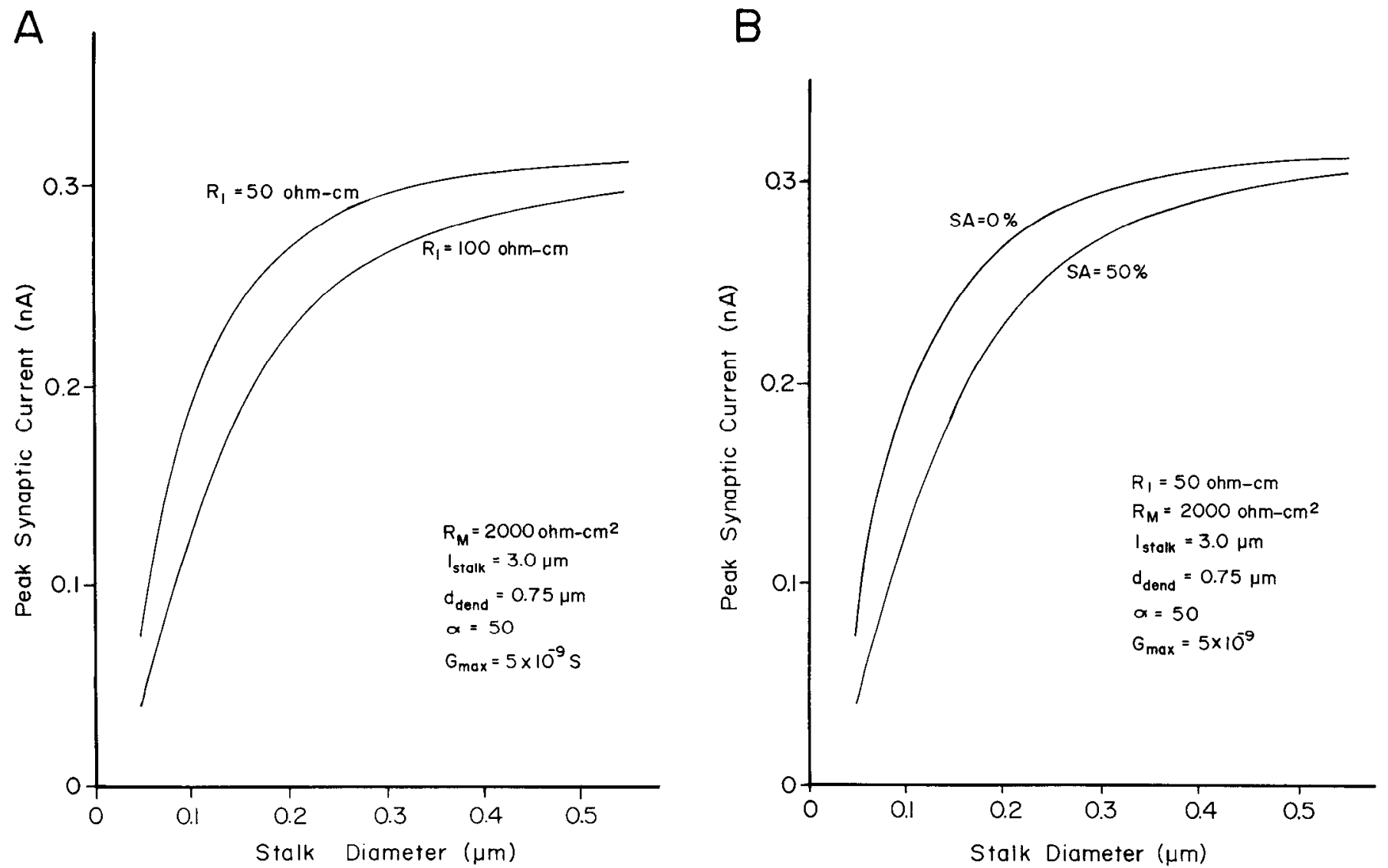

Figure 8. Dependence of the current attenuating effect of dendritic spines on intracellular resistivity $(A)$ and relative volume occupied by the spine apparatus $(B)$. In $A$ the effect of spine stalk diameter for a long spine is shown using two values for the intracellular resistivity. The effect is approximately the same as changing stalk length. A similar result is obtained if the spine apparatus occupies a proportion of the volume of the spine stalk $(B)$.

resistance. For larger stalk diameters in Figure $8 A$ the dendrite becomes a measurable influence, and there was a small deviation from the simple relationship with length. Electrotonic lengths of spine stalks are proportional to the square root of their cytoplasmic resistivities, and the length of a spine required to produce an important attenuation of synaptic currents is thus dependent upon the resistance of the cytoplasm. Anything that selectively increased the resistivity of the spine stalk cytoplasm would be effectively increasing the length of the spine. A large proportion of some spines, and probably some proportion of all dendritic spines, is taken up by the enclosed space of a membranous organelle, the spine apparatus (Gray, 1959). In some cases it may occupy well over half of the volume of the spine stalk, and in places it may nearly occlude the cytoplasmic space. Although it may have other functions (e.g., Wilson et al., 1983), it is almost certainly an obstacle to intracellular current flow. Although this has not been demonstrated directly, it may be inferred from the complete enclosure of the interior compartment of the spine apparatus by a single continuous membrane separating it from the current-carrying portion of the cytoplasm. Its presence will thus be expected to increase the intracellular resistivity and the effective length of the spine stalk, and it will do this to a degree depending upon the proportion of the spine neck volume it occupies. This effect is shown in Figure $8 B$ in which a moderately large spine apparatus occupying half the volume of the spine stalk was simulated as a doubling of the intracellular resistivity of the stalk. No such effect was obtained using specific increases in cytoplasmic resistivity of spine heads or dendrites to simulate the presence of the spine apparatus or other membranous organelles in these compartments.

Membrane resistivity. The membrane resistivity of dendritic neurons is determined from experimental measurement of the whole neuron resistance. This value is usually derived from voltage deflections produced by low-amplitude, long-duration current pulses applied into the soma from an intracellular electrode. To extract membrane resistivity from the whole neuron input resistance, an electrotonic model of the neuron must be constructed, and the estimate of membrane resistivity will be no more accurate than the model. The input resistance of neostriatal spiny neurons ranges from 10 to 20 megohms as measured in vivo in cat (Sugimori et al., 1978) and rat (C. J. Wilson, unpublished observations) and in rat neostriatal slices in vitro ( $\mathrm{T}$. Kita, H. Kita, and S.T. Kitai, personal communication). Using an isopotential soma $15 \mu \mathrm{m}$ in diameter and six dendritic trees treated as $2.5-\mu \mathrm{m}$ passive uniform cables of infinite 
length, Sugimori et al. (1978) arrived at an estimate of $8000 \mathrm{ohm}-\mathrm{cm}^{2}$ for the membrane resistivity of these cells. Repeating those authors' calculations but using dendrites terminated at a distance of $200 \mu \mathrm{m}$ from the soma yields a value of about $1600 \mathrm{ohm}-\mathrm{cm}^{2}$. Similar estimates are obtained using the model favored here, in which the proximal branch points are ignored and the neuron is considered to have about 25 dendrites of $0.75 \mu \mathrm{m}$ diameter. Probably it is only safe at this point to conclude that the neostriatal spiny neuron has a membrane resistivity between 1000 and 8000 ohm- $\mathrm{cm}^{2}$.

If a passive cable is terminated by a high resistance, increasing its membrane resistance increases its input resistance both by increasing its length constant (and so bringing that high resistance effectively closer to the input) and by decreasing the leakage current across its membrane. If a dendrite is terminated by a lower resistance, increasing its membrane resistance may have a much less pronounced effect, since it is bringing that low resistance electrotonically closer to the input, despite the decreased leakage across the membrane. The spine stalk is terminated by the lower impedance of the dendrite. Both the dendritic and the spine stalk leakage currents are decreased with increased membrane resistance. The dendritic input impedance is increased, but the spine stalk is effectively shortened. These two effects oppose each other. The net result, as shown in Figure 9, is that membrane resistivity has very little influence on the input impedance at axospinous synapses or on the synaptic currents generated by those synapses. The peak synaptic current generated at an axodendritic synapse is also shown in Figure 9, and it is also relatively unaffected by membrane resistivity over the range used in these calculations. In this case, however, it is because the dendritic synaptic potentials are small enough that synaptic current is independent of input impedance.

It should be mentioned that in these simulations the conductance waveform was modified for each value of membrane resistivity to take into account the effect of membrane resistance on the time constant. This was required because the conductance waveform used in these simulations was defined in units of the time constant. To keep the duration of simulated synaptic action constant in absolute time units, alpha was increased and decreased by the same proportion as the membrane resistance (using $\alpha=50$ at a membrane resistivity of 2000 ohm- $\mathrm{cm}^{2}$ as the starting point). What evidence is available indicates that the time course of synaptic conductance does not co-vary with membrane time constant of the postsynaptic cell (e.g., Jack et al., 1971).

Dendritic diameter. For dendritic spines to attenuate synaptic current beyond that which would occur at an axodendritic synapse, the spine stalk must substantially increase the input impedance at the synaptic site. This will necessarily depend to some degree upon the dendrite as well as the spine. For the infinitely long dendrite, the only parameter not already considered is cross-sectional diameter. In Figure 10 the effect of dendritic diameter on synaptic current is shown for an axodendritic synapse and for three different dendritic spines. In all cases synaptic current increased with dendritic diameter over a range of small diameters, after which further increases

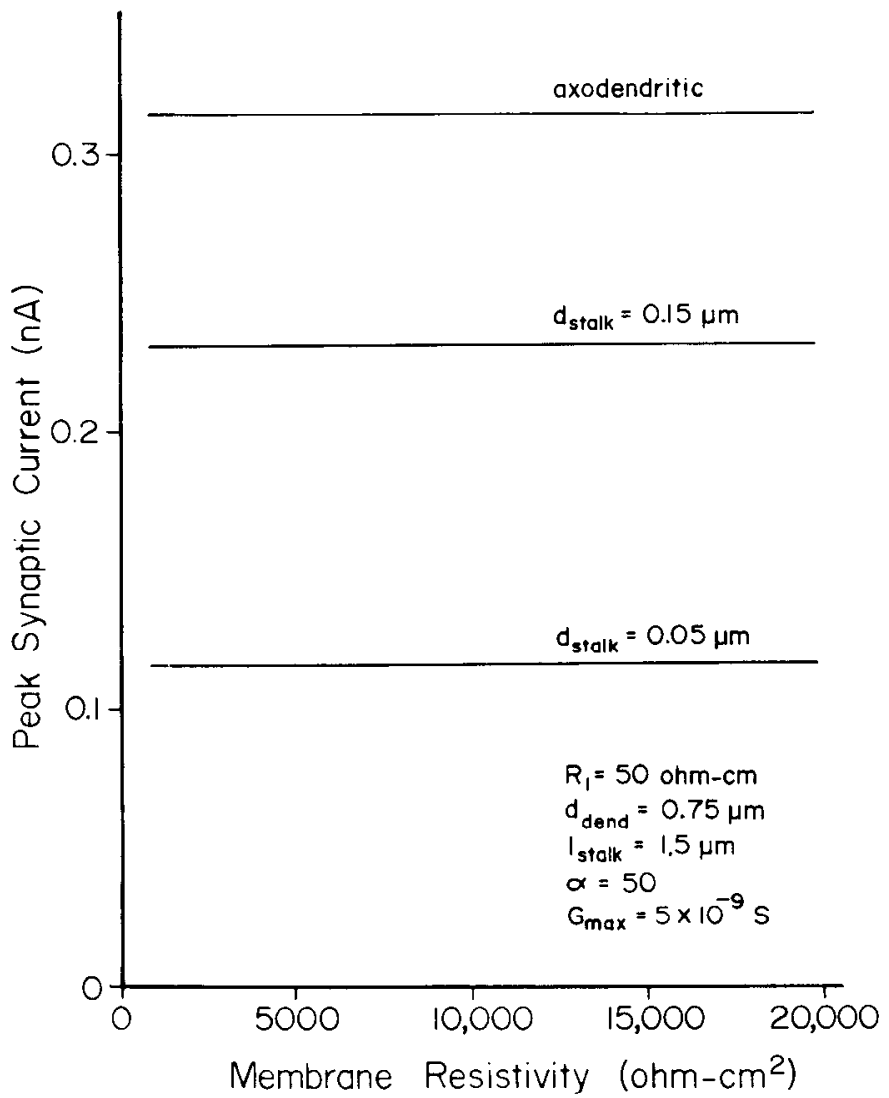

Figure 9. Relative independence of the current attenuating effect of dendritic spines and membrane resistivity. The current attenuating effect of dendritic spines is represented by the distance between the curves. The time course of the synaptic conductance change was adjusted to be constant in absolute time units, using $\alpha=50$ and a membrane resistivity of 2000 $\mathrm{ohm}-\mathrm{cm}^{2}$ as a standard.

had relatively little effect. Dendritic diameter always had the greatest effect on axodendritic synapses, and the maximal synaptic current in these occurred when dendrites were large enough that synaptic potentials were small compared to the driving potential and so the synapses behaved linearly. Axospinous synapses were less dependent upon the dendritic diameter and reached their maximum synaptic current with smaller dendrites. In their cases the maximal current occurred when the dendritic input impedance became too small to contribute significantly to that imposed by the spine stalk. The current attenuating effect of the spines, represented by the divergence of the curves in Figure 10, was already well developed for dendrities $0.25 \mu \mathrm{m}$ in diameter and was relatively unaffected by increases in dendritic diameter beyond about $1.0 \mu \mathrm{m}$.

Dendritic termination. The length of the postsynaptic dendrite and its mode of termination were not treated analytically in the model used here. The relative independence of axospinous synaptic currents from dendritic diameter and the large difference between the time courses of charge distribution on the spine and dendrite allowed an approximation of the effects of dendritic termination, however. For dendrites of sufficient length that redistribution of charge on the spine is much more 
rapid than redistribution on the dendrite, the terminated dendrite solutions of Jack and Redman (1971) can be used as asymptotes for the characteristic responses. The resulting characteristic responses are good approximations to the responses for spines on similarly terminated dendrites. For spines and dendrites in the size ranges used here, such approximations were found to be possible for dendrites of $50 \mu \mathrm{m}$ length or longer.

Using the characteristic responses obtained as just described, the effects of dendritic termination were studied for spines placed in one or two dendrites ranging in length from $50 \mu \mathrm{m}$ to infinity. Dendritic length over this range had little appreciable effect on synaptic currents at axospinous synapses. Less than a $5 \%$ error occurred when the infinite dendrite was used to estimate peak synaptic or base currents fro the three spine sizes used in Figure 10 placed midway along a $100-\mu \mathrm{m}$ dendrite with insulated terminations. A less than $10 \%$ error in peak currents was obtained for a spine placed on one end of a single $50-\mu \mathrm{m}$ dendrite with an insulated termination at the other end. Thus, the model presented here is valid for a wide range of spine shapes and dendritic arrangements, and it can be shown to hold as long as the spine is attached to at least one dendritic segment with a diameter of $0.25 \mu \mathrm{m}$ or greater and a length of at least $50 \mu \mathrm{m}$.

Effect at the soma. The independence of axospinous synaptic currents from the subtleties of branching and

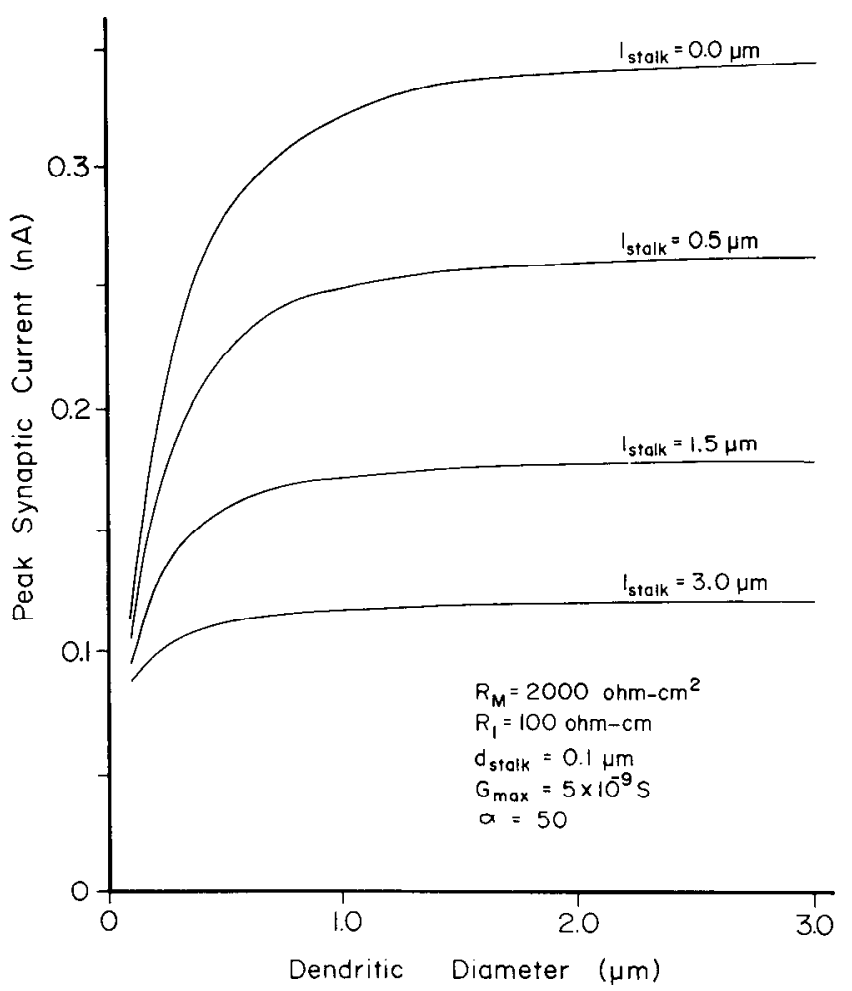

Figure 10. Effect of dendritic diameter on synaptic current at axodendritic (uppermosl curve) and axospinous (lower three curves) synapses. The current attenuating effect of spines is represented by the distance between these curves. Dendritic diameter becomes relatively unimportant with dendrites over $0.5 \mu \mathrm{m}$ in diameter. termination of the dendritic tree does not extend to the postsynaptic potentials generated by those currents. The spine base synaptic potentials, for example, varied greatly in the above decribed simulations with changes in dendritic diameter and termination conditions that did not produce any appreciable change in the current injected into the dendrite. In some cases those postsynaptic potentials may be of interest, but usually it is the potential at the cell body that is of primary concern. For spines that do effectively control synaptic current independently of the dendritic conditions, it is possible to simulate the effect of axospinous synaptic activation on any neuron model using this synaptic current as the input. The results from a variety of spine shapes can be directly compared to axodendritic synaptic effects in the same neuron model. Figure $11 A$ shows an example using the dendritic neuron model introduced by Rinzel and Rall (1974) with zero order of branching and parameters set appropriately for neostriatal spiny neurons. The distance between the axospinous synapse and the origin of the dendrites was varied to examine the effect of position in the dendritic field. The changes in PSP waveform at the soma were similar to those expected for any brief current injected into models of this sort (Rinzel and Rall, 1974). Shape indices of axospinous synapses did indicate a more distal location for these synapses than for axodendritic synapses at the same distance from the soma, but otherwise all contacts produced similar PSP waveforms. The conductance changes were the same in all these simulations, and the small changes in waveform that were attributable to the spine were due to a slowing of the current injection into the dendrite compared to that occurring at axodendritic synapses.

The results of simulations with the Rinzel and Rall model comparing three spine shapes and axodendritic synapses at various locations are shown in Figure $11 B$. The current attenuating effect of dendritic spines produced differences in somatic EPSP amplitudes at all locations on the dendrites. The magnitude of these differences was comparable to that produced by position in the dendritic field.

A second effect of dendritic spines is also illustrated in Figure $11 B$. Because they isolated their synapses from the input impedance of the dendrite, they decreased the extent to which synaptic currents depended upon synaptic location. For axodendritic synapses, two different mechanisms contributed to the reduction of somatic EPSP amplitudes observed as the synapse was placed on more distal dendritic locations. One of these was the decrement of responses produced by applied currents through redistribution of charge along the membrane and by leakage currents. This mechanism also attenuated axospinous EPSPs as they were conducted to the soma. The second was a current attenuation due to the increased input impedance of dendrites at synaptic sites more distant from the soma (e.g., MacGregor, 1968; Rall and Rinzel, 1973). This second factor did not act on axospinous synapses. The difference can be seen in Figure $11 B$ by the relatively constant amplitude of axospinous EPSPs beyond about $80 \mu \mathrm{m}$ from the soma and continuing to the end of the dendrite. This effect would be even more pronounced in more complex neuronal 

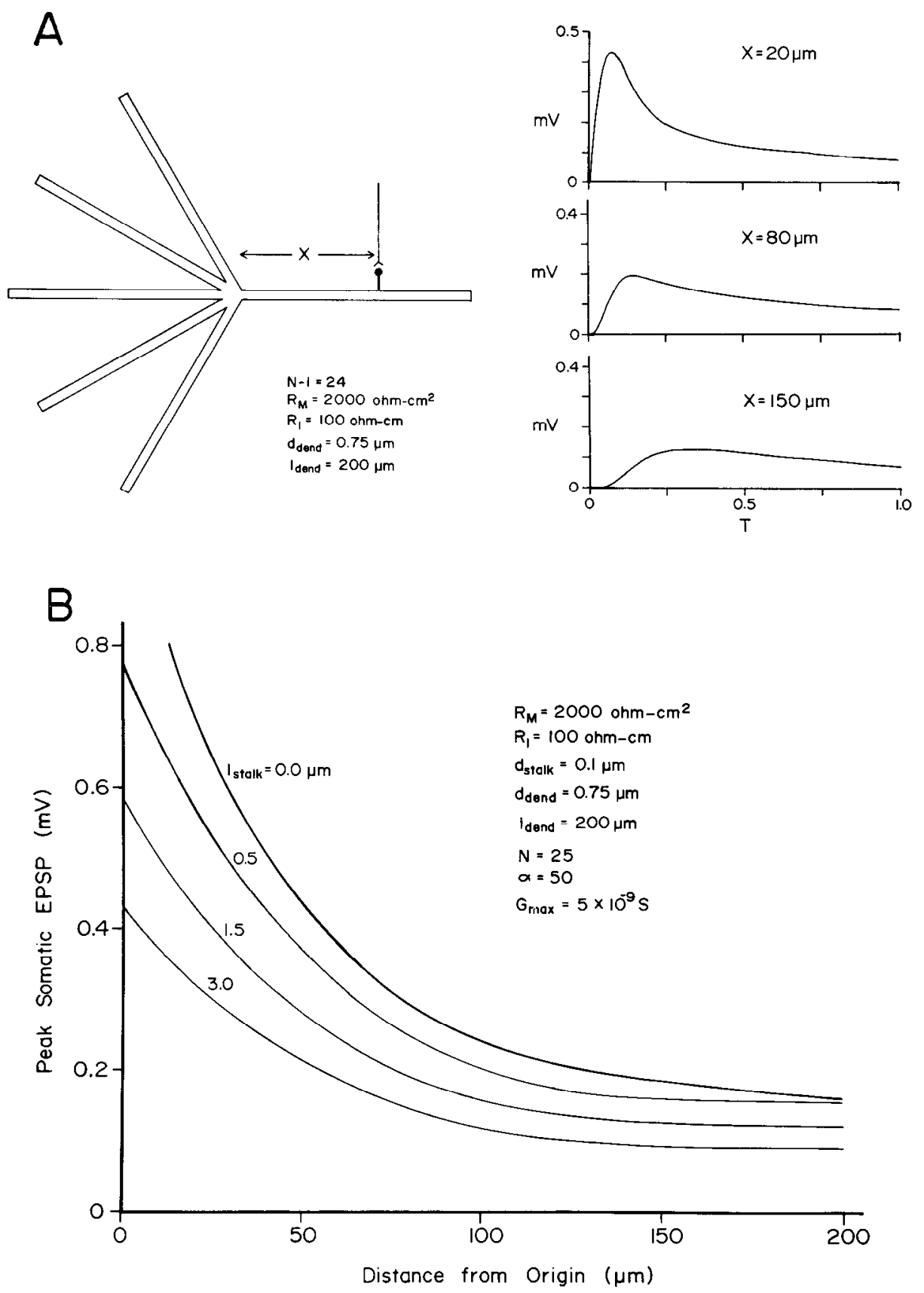

Figure 11. A, Application of spine-generated synaptic currents to a dendritic neuron model. The Rinzel and Rall neuron model was used to simulate a neostriatal spiny neuron with 25 dendrites of $200 \mu \mathrm{m}$ length and one axospinous synapse located on one dendrite at a variable distance from the soma. The somatic EPSPs generated at three different distances from the soma are shown at the right. Their time courses are similar to those expected for any transient current injection. The spine in this case was the $1.5 \mu \mathrm{m}$ length spine in $B . B$, The peak somatic EPSPs generated by the model illustrated in $A$ for three different spines and an axodendritic synapse. The effect of spine shape is apparent at all distances from the soma. For neostriatal neurons, dendritic spines and excitatory synapses are excluded from the first approximately $25 \mu \mathrm{m}$ of dendrite. This is the region in which synaptic location is most important.

models with tapering dendrites or higher orders of branching. The result was a de-emphasis of synaptic location in the dendritic tree as a weighting factor of axospinous synapses. Axospinous synapses on somata and very proximal dendrites retained their powerful ef- fect on the somatic membrane potential, but synapses on spines located in the midportion or distal portion of the dendritic field became about equally weighted. For neurons that exclude axospinous and axodendritic excitatory synapses from their most proximal dendritic re- 
gions (as occurs in neostriatum and many other areas), the effect of spine size and shape is further emphasized over that of synaptic location.

\section{Discussion}

Questionable assumptions. Linear cable models like the one presented here rely heavily on the assumptions of linearity and uniformity of dendritic membrane. Both assumptions have been seriously challenged by recent ncurophysiological findings (Spencer and Kandel, 1961; Anderson and Lomo, 1966; Llinás and Nicholson, 1971; Schwartzkroin and Slawsky, 1977; Traub and Llinás, 1979; Wong et al., 1979; Llinás and Sugimori, 1980 a, b). Until the membrane properties of individual dendritic spines can be measured directly, the validity of these assumptions must be in doubt. This is especially acute for dendritic spines because of the finding that large amplitude fast transient EPSPs are expected to occur on the synaptic membrane. Such large displacements of the membrane potential are likely to evoke active currents in neuronal membrane capable of generating them. The possible existence of action potentials in dendritic spines has been suggested by Diamond et al. (1970), who recorded spike-like activity from their intermediate unit, believed to be a dendritic spine on a motoneuron. It is possible, of course, that such a signal could in fact be a fast spike-like EPSP as predicted by the passive model. If action potentials do occur on dendritic spines but do not spread down the stalk or to the dendrite (because of the lower dendritic input impedance), they should behave in a manner very similar to that described here for synaptic potentials. Currents generated by active conductances should be attenuated in the same way as those of synaptic conductances. The same temporal and spatial parameters of the spine and the conductance change will determine the current injected into the dendrite.

The results of the present model also indicate that nonuniformity of the linear membrane and cytoplasmic parameters will produce predictable quantitative changes in the behavior of the system without altering its general form. Some deviations from uniformity are expected from morphological findings and so are of special interest. The possible effect of the spine apparatus on the intracellular resistance in the spine stalk is one of these. If this organelle is subject to changes in size or position in the spine, these changes may be expected to produce large changes in synaptic transmission at that location. Those changes would probably be mistakenly attributed to a change in transmitter release or receptor sensitivity. Spatial nonuniformity of membrane resistance on the spine stalk could also have a profound effect on the degree to which spines attenuate synaptic current. Especially interesting is the possibility that membrane resistance may be locally affected by substances released in conjunction with neurotransmitters or diffusing in the extracellular space. Spine stalks are electrotonically shortened or lengthened by changes in membrane resistance. Changes in the effectiveness of synaptic transmission due to the action of membrane resistance modulating substances will also be misinterpreted if the cable properties of dendritic spines are ignored in the analysis.

Synaptic interactions. One known situation in which the membrane resistance of the spine is sure to undergo radical and reversible changes is that in which an inhibitory synapse is present on the spine stalk. This occurs on about $10 \%$ of spines in rat neostriatum (Wilson et al., 1983). Most of the inhibitory synapses probably arise from axon collaterals of the spiny cells themselves, which make nearly half of their intrastriatal synaptic contacts onto spine stalks (Wilson and Groves, 1980). 'They have also been reported in cerebral cortex (Collonier, 1968; Jones and Powell, 1969). In other structures still more complex synaptic arrangements are common. In rat locus ceruleus, for example, somatic and dendritic spines often have a number of synapses and may receive contacts of all morphologial types found in that nucleus (Tatemichi and Ramon-Moliner, 1975; Groves and Wilson, 1980). Axosomatic synapses are also preferentially located around the bases of somatic spines in this structure (Groves and Wilson, 1980). These arrangements make possible very complicated synaptic interactions. Synapses located on the same spine will interact in two ways. The large postsynaptic potentials generated by small synaptic currents from one contact will have a dramatic effect on the voltage gradient driving currents at the other synapses (MacGregor, 1968). This type of nonlinear synaptic interaction will also occur between axospinous and axodendritic synapses and between synapses on separate spines. This is because membrane potential displacements in the dendrite are conducted into the spine (in the opposite direction to that of axospinous synapses) with little attenuation. This topic has been addressed by Shepherd and Brayton (1979). The second type of synaptic interaction is one in which a synaptic conductance at one site alters the input impedance at another synapse. Excitatory synapses on separate dendritic spines will be relatively isolated from each other in this regard, being separated by two high-resistance spine necks and a low-resistance dendrite. Llinás and Hillman (1969) compared this arrangement to a constant current source, in which the constant voltage is supplied by the reversal potential of the synapse and the current limiting resistor by the spine stalk resistance. Over a wide range of spine shapes and electrical parameters, this analogy appears to provide a good approximation to dendritic spine properties. Those same authors suggested that this would increase the linearity of summation of axospinous excitatory synaptic potentials by the dendrite. By the same argument, dendritic inhibition will be even more effective in blocking axospinous excitation. The current injected into the dendrite by axospinous synapses will not be increased by the action of a shunting inhibitory conductance on the dendrite. Nonetheless, the inhibitory conductance will very effectively shunt the axospinous excitatory current.

'The relative time courses of the synaptic conductance changes are very important in determining both the electrotonic properties of individual axospinous synapses and the ways in which synapses on the same spine will interact. The magnitude of the current attenuating effect of the spine depends upon the duration of the synaptic conductance being longer than or comparable to the time required for charge redistribution on the spine, but much shorter than that required for charge equalization on the 
dendrite. For such fast conductance changes, the spine will represent a much larger impedance than the dendrite. For longer conductance changes, the dendrite becomes an important factor and the spine is de-emphasized. Thus, a very slowly acting synaptic conductance will not be much affected by the presence of a spine in comparison to a fast one at the same location. Slow conductance changes may accompany rapid synaptic transmission at single synapses by a number of mechanisms (Libet and Toska, 1969; Kehoe, 1972; Jan et al., 1979; Adams and Brown, 1980; Brown et al., 1981). If such events occur at axospinous synapses, the two conductance changes may act as though they were occurring at radically different synaptic locations.

Spine shape and size. The possibility that the effectiveness of synapses might be altered by ongoing modification of cell shape was suggested by Rall (1962) and by MacGregor (1968) on the basis of their calculations of cable properties of dendritic neurons. Because of the square root relationship between dendritic diameter and electrotonic length, small changes in dendritic diameter will have their greatest effect when involving smallcaliber dendrites. Dendritic terminals are the most desirable locus for a selectively changeable element, to allow changes in one or only a few related synapses with minimal effect on other contacts. As pointed by Rall (1974), these considerations make the dendritic spine an ideal variable dendritic element for the selective alteration of the effectiveness of individual synapses. Since this suggestion was first made, a number of authors have reported changes in dendritic spines under natural or near natural conditions (Fifková and Van Harreveld, 1977; Coss and Globus, 1978; Bradley and Horn, 1979; Burgess and Coss, 1980; Coss et al., 1980; Brandon and Coss, 1982) and in some cases these can be associated with a change in synaptic transmission (Van Harreveld and Fifková, 1975; Fifková and Van Harreveld, 1977; Fifková and Anderson, 1981). The simulations presented here indicate that differences in spine shape and size of the magnitude and type described by these authors are sufficient to produce substantial changes in the effectiveness of synaptic transmission.

\section{Appendix}

\section{Characteristic responses}

The Laplace transform input impedance at the spine head and the transfer functions for voltage and current at the spine base for the arrangement shown in Figure 1 can be shown by conventional (see Jack et al., 1975) means to be

$$
\begin{aligned}
& \frac{\bar{V}_{\text {head }}}{\bar{I}_{\text {syn }}}=\frac{\frac{R_{\text {head }}}{q(q+a)}\left(1-b e^{-\left[2 q L_{\text {stall }}\right]}\right)}{1-\left(\frac{q-a}{q+a}\right) b e^{-\left[2 q L_{\text {stall }}\right]}} \\
& \frac{\bar{V}_{\text {base }}}{\bar{I}_{\text {syn }}}=\frac{\frac{R_{\text {head }}}{q(q+a)}(1-b) e^{-\left[q L_{\text {stalik }}\right]}}{1-\left(\frac{q-a}{q+q}\right) b e^{-\left[2 q L_{\text {stalik }}\right]}}
\end{aligned}
$$

$$
\frac{\bar{I}_{\text {base }}}{\bar{I}_{\text {gyn }}}=\frac{\frac{a(1+b)}{q+a} e^{-\left\{q L_{\text {stallk }}\right]}}{1-\left(\frac{q-a}{q+a}\right) b e^{-\left[2 q L_{\text {stalk }}\right]}}
$$

where $a$ and $b$ are defined as under "Materials and Methods" and $q=(s+1)^{1 / 2}$. Expanding the denominators into infinite series and solving for the Laplace transform of the voltages and currents gives

$$
\begin{aligned}
\bar{V}_{\text {head }}=\bar{I}_{\text {syn }} R_{\text {head }} & {\left[\sum_{n=0}^{\infty} \frac{(q-a)^{n}}{q(q+a)^{n+1}} b_{n} e^{-\left[2 n q L_{\text {stalk }}\right]}\right.} \\
& \left.-\sum_{n=0}^{\infty} \frac{(q-a)^{n}}{q(q+a)^{n+1}} b^{n+1} e^{-\left[2(n+1) q L_{\text {stalk }}\right)}\right] \\
\bar{V}_{\text {base }}= & \bar{I}_{\text {syn }} R_{\text {head }}(1-b) \sum_{n=0}^{\infty} \frac{(q-a)^{n}}{q(q+a)^{n+1}} b^{n} e^{-\left[(2 n+1) q L_{\text {stall }}\right]} \\
\bar{I}_{\text {base }} & =\bar{I}_{\text {syn }} a(1-b) \sum_{n=0}^{\infty} \frac{(q-a)^{n}}{(q+a)^{n+1}} b^{n} e^{-\left\{(2 n+1) q L_{\text {stalk }}\right]}
\end{aligned}
$$

The two transform pairs that are required can be obtained as described by Jack and Redman (1971). They are:

$$
\frac{\left(s^{1 / 2}-a\right)^{n} e^{-P s^{1 / 2}}}{s^{1 / 2}\left(s^{1 / 2}+a\right)^{n+1}} \Leftrightarrow \frac{e^{-\left[p^{2} / 4 T\right]}}{(\pi / 2)^{1 / 2}} \sum_{r=0}^{n} \frac{(-n)_{r}}{r !}(2 a)^{r}(2 T)^{r / 2} e^{\left[w^{2} / 4\right]} D_{-(r+1)}[w]
$$

and

$$
\begin{aligned}
& \frac{\left(s^{1 / 2}-a\right)^{n} e^{-P_{s}^{1 / 2}}}{\left(s^{1 / 2}+a\right)^{n+1}} \Leftrightarrow \frac{e^{\left.-\mid \mu^{2 / 4} / 4 T\right]}}{(\pi T)^{1 / 2}}\left[\sum_{r=0}^{n} \frac{(-n)_{r}}{r !}(2 a)^{r}(2 T)^{r / 2}(r\right. \\
& \quad+1) e^{\left[w^{2} / 4\right]} D_{-(r+2 w)}[w]+\sum_{r=0}^{n} \frac{(-n)_{r}}{r !}(2 a)^{r}(2 T)^{r-1 / 2} P e^{\left[\omega^{2} / 4\right]} D_{-(r+1)}[w]
\end{aligned}
$$

where $W=2^{1 / 2}\left(a T^{1 / 2}+\frac{P}{2 T^{1 / 2}}\right)$ and $D_{\nu}[z]$ is the parabolic cylinder function.

Applying the transform pairs and the shift theorem to equations $4 \mathrm{~A}, 5 \mathrm{~A}$, and $6 \mathrm{~A}$ and using an impulse current at the spine head as input gives the characteristic responses (equations 1,2, and 3 under "Materials and Methods").

\section{Special cases}

1. Spine head is vanishingly small, $R_{\text {head }}=\infty$. Equations $1 \mathrm{~A}$ to $3 \mathrm{~A}$ become:

$$
\begin{gathered}
\frac{\bar{V}_{\text {head }}}{\bar{I}_{\text {syn }}}=\frac{R_{\text {stalkoo }}\left(1-b e^{-\left[2 q L_{\text {stalk }}\right]}\right)}{q\left(1+b e^{-\left\{2 q L_{\text {stallt }}\right]}\right)} \\
\frac{\bar{V}_{\text {base }}}{\bar{I}_{\text {syn }}}=\frac{R_{\text {stalk }}(1-b) e^{-\left[q L_{\text {stalk }}\right]}}{q\left(1+b e^{-\left[2 q L_{\text {stalk }}\right]}\right)} \\
\frac{\bar{I}_{\text {base }}}{\bar{I}_{\text {syn }}}=\frac{(1+b) e^{-\left[q L_{\text {stallk }}\right]}}{\left(1+b e^{-\left[2 q L_{\text {stalk }}\right]}\right)}
\end{gathered}
$$

Expanding the denominator of these gives:

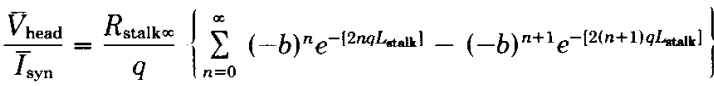

$$
\begin{aligned}
& \frac{\bar{V}_{\text {hase }}}{\bar{I}_{\text {syn }}}=\frac{R_{\text {stalkos }}}{q}(1-b) \sum_{n=0}^{\infty}(-b)^{n} e^{-\left[(2 n+1) q L_{\text {salk }}\right]}
\end{aligned}
$$


The Journal of Neuroscience

Dendritic Spine Model

295
The transform pairs required for these are:

$$
\frac{e^{-K s^{1 / 2}}}{s^{1 / 2}} \Leftrightarrow \frac{1}{(\pi T)^{1 / 2}} e^{-\left[K^{2} / 4 T\right]}
$$

and

$$
e^{-K_{v} / 2} \Leftrightarrow \frac{K}{(2 T)(\pi T)^{1 / 2}} e^{-\left[K^{2} / 4 T\right]}
$$

Applying the shift theorem and these transform pairs to equations $10 \mathrm{~A}$ to $12 \mathrm{~A}$ and using an impulse synaptic current gives equations 4 to 6 under "Materials and Methods."

2. Spine stalk is vanishingly short. With $L_{\text {stalk }}=0$, equations $1 \mathrm{~A}$ to $3 \mathrm{~A}$ become:

$$
\begin{gathered}
\frac{\bar{V}_{\text {head }}}{\bar{I}_{\text {syn }}}=\frac{\bar{V}_{\text {base }}}{\bar{I}_{\text {syn }}}=\frac{R_{\text {head }}}{q\left(q+\frac{a(1+b)}{(1-b)}\right)} \\
\frac{\bar{I}_{\text {hase }}}{\bar{I}_{\text {syn }}}=\frac{a \frac{(1+b)}{(1-b)}}{q+a \frac{(1+b)}{(1-b)}}
\end{gathered}
$$

The required transform pairs are:

$$
\begin{gathered}
\frac{1}{s^{1 / 2}\left(s^{1 / 2}+K\right)} \Leftrightarrow e^{K^{2} T} \operatorname{erfc}\left[K(T)^{1 / 2}\right] \\
\frac{1}{s^{1 / 2}+K} \Leftrightarrow \frac{1}{(\pi T)^{1 / 2}}-K e^{K^{2} T} \operatorname{erfc}\left[K(T)^{1 / 2}\right]
\end{gathered}
$$

These, together with the shift theorem, give the characteristic responses.

$$
\begin{aligned}
& V_{\text {head }}^{*}=V_{\text {base }}^{*}=R_{\text {head }} e^{-T} e^{\rho^{2 T}} \operatorname{erfc}\left[\rho(T)^{1 / 2}\right] \\
& I_{\text {hase }}^{*}=\rho e^{-T}\left[\frac{1}{(\pi T)^{1 / 2}}-\rho e^{\rho^{\rho^{2} T}} \operatorname{erfc}\left[\rho(T)^{1 / 2}\right]\right]
\end{aligned}
$$

where $\rho=\frac{2 R_{\text {head }}}{R_{\text {dend } \infty}}=a \frac{(1+b)}{(1-b)}$.

3. Asymptote as $T$ becomes large. Expansion of the exponential terms in Equations 1A to $3 \mathrm{~A}$ to infinite series gives:

$$
\begin{gathered}
\frac{\bar{V}_{\text {head }}}{\bar{I}_{\text {syn }}}=\frac{\frac{R_{\text {head }}}{q(q+a)}\left[1-b\left(1-2 q L_{\text {stalk }}+\frac{\left(2 q_{\text {stalk }}\right)^{2}}{2 !}-\cdots\right)\right]}{1-\left(\frac{q-a}{q+a}\right) b\left(1-2 q L_{\text {stalk }}+\frac{\left(2 q L_{\text {stalk }}\right)^{2}}{2 !}-\cdots\right)} \\
\frac{\bar{V}_{\text {base }}}{\bar{I}_{\text {syn }}}=\frac{\frac{q(q+a)}{1-\left(\frac{q-a}{q+a}\right) b\left(1-2 q L_{\text {stalk }}+\frac{\left(2 q L_{\text {stalk }}\right)^{2}}{2 !}-\cdots\right)}}{\frac{a(1+b)}{(q+a)}\left(1-q L_{\text {stalk }}+\frac{\left(q L_{\text {stalk }}\right)^{2}}{2 !}-\cdots\right)} \\
\frac{\bar{I}_{\text {base }}}{\bar{I}_{\text {syn }}}=\frac{1-\left(1-q L_{\text {stalk }}+\frac{\left(q L_{\text {stalk }}\right)^{2}}{2 !}-\cdots\right)}{1-\left(\frac{q-a}{q+a}\right) b\left(1-2 q L_{\text {stalk }}+\frac{\left(2 q L_{\text {stalk }}\right)^{2}}{2 !}-\cdots\right)}
\end{gathered}
$$

Let $q=s^{1 / 2}$. As time proceeds, $q^{2}$ approaches zero.
Rearranging terms and setting $q^{2}=0$ wherever it appears gives:

$$
\frac{\bar{V}_{\text {head }}}{\bar{I}_{\text {syn }}}=\frac{\frac{R_{\text {head }}}{1+a L_{\text {stalk }}}}{q\left(q+\frac{a(1+b)}{(1-b)\left(1+a L_{\text {stalk }}\right)}\right)}
$$

$$
\begin{gathered}
+\frac{\frac{R_{\text {head }} L_{\text {stalk }}(1+b)}{(1-b)\left(1+a L_{\text {stalk }}\right)}}{q+\frac{a(1+b)}{(1-b)\left(1+a L_{\text {stalk }}\right)}} \\
\frac{\bar{V}_{\text {base }}}{\bar{I}_{\text {syn }}}=\frac{\frac{R_{\text {head }}}{1+a L_{\text {stalk }}}}{q\left(q+\frac{a(1+b)}{(1-b)\left(1+a L_{\text {stalk }}\right)}\right)} \\
\frac{\bar{I}_{\text {base }}}{\bar{I}_{\text {syn }}}=\frac{\frac{a(1+b)}{\left(1+a L_{\text {stalk }}\right)(1-b)}}{q+\frac{a(1+b)}{(1-b)\left(1+a L_{\text {stalk }}\right)}}
\end{gathered}
$$

These may be inverted using the transform pairs given above. Using the shift theorem to give the result for $q=$ $(s+1)^{1 / 2}$,

$$
\begin{aligned}
& V_{\text {head }}^{*}=\frac{R_{\text {head }}}{1+a L_{\text {stalk }}} e^{-T} e^{y^{2} T} \operatorname{erfc}\left[y(T)^{1 / 2}\right]
\end{aligned}
$$

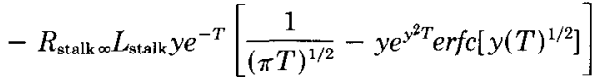

$$
\begin{aligned}
& V_{\text {base }}^{*}=\frac{R_{\text {head }}}{1+a L_{\text {stalk }}} e^{-T} e^{y^{2} T} \operatorname{erfc}\left[y(T)^{1 / 2}\right] \\
& I_{\text {base }}^{*}=y e^{-T}\left[\frac{1}{(\pi T)^{1 / 2}}-y e^{y^{2} T} \operatorname{erfc}\left[y(T)^{1 / 2}\right],\right.
\end{aligned}
$$

where

$$
y=\frac{a(1+b)}{(1-b)\left(1+a L_{\text {stalk }}\right)}
$$

With the equalization of charge in the spine, the second term in the head response becomes negligible. It may be noted that when stalk length is zero, these reduce to the exponential long time asymptote of Jack and Redman (1971).

\section{References}

Abramowitz, M., and I. A. Stegun (1970) Handbook of Mathematical Functions, Dover, New York.

Adams, P. R., and D. A. Brown (1980) Lutenizing hormonereleasing factor and muscarinic agonists act on the same voltage-sensitive $\mathrm{K}$-current in bullfrog sympathetic neurones. Br. J. Pharmacol. 68: 353-355.

Andersen, P., and T. Lomo (1966) Mode of activation of hippocampal pyramidal cells by excitatory synapses on dendrites. Exp. Brain Res. 2: 247-260.

Barrett, J. N., and W. E. Crill (1974) Influences of dendritic location and membrane properties on the effectiveness of synapses on cat motoneurones. J. Physiol. (Lond.) 239: 325345.

Bradley, P., and G. Horn (1979) Neuronal plasticity in the chick brain: Morphological effects of visual experience on neurones in the hyperstriatum accessorium. Brain Res. 162: 148-153. 
Brandon, J. G., and R. G. Coss (1982) Rapid dendritic spine stem shortening during one-trial learning: The honeybee's first orientation flight. Brain Res. 252: 51-61.

Brown, D. A., A. Constanti, and P. R. Adams (1981) Slow cholinergic and peptidergic transmission in sympathetic ganglia. Fed. Proc. 40: 2625-2630.

Burgess, J. W., and R. G. Coss (1980) Crowded jewel fish show changes in dendritic spine density and spine morphology. Neurosci. Lett. 17: 277-281.

Chang, H. -T. (1952) Cortical neurons with particular reference to the apical dendrites. Cold Spring Harbor Symp. Quant. Biol. 17: 189-202.

Colonnier, M. (1968) Synaptic patterns on different cell types in the different laminae of the cat visual cortex. Brain Res. 9: 268-287.

Coss, R. G., and A. Globus (1978) Spine stems on tectal interneurons in jewel fish are shortened by social stimulation. Science 200: 787-790.

Coss, R. G., J. G. Brandon, and A. Globus (1980) Changes in morphology of dendritic spines on honeybee calycal interneurons associated with cumulative nursing and foraging experiences. Brain Res. 192: 49-60.

Diamond, J., E. G. Gray, and G. M. Yasargil (1970) The function of the dendritic spine: An hypothesis. In Excitatory Synaptic Mechanisms, P. Andersen and J. K. S. Jansen, eds., pp 213-222. Universitet Forlaget, Oslo.

Fifková, E., and C. L. Anderson (1981) Stimulation-induced changes in dimensions of stalks of dendritic spines in the dentate molecular layer. Exp. Neurol. 74: 621-627.

Fifková, E., and A. Van Harreveld (1977) Long-lasting morphological changes in dendritic spines of dentate granular cells following stimulation of the entorhinal area. J. Neurocytol. 6: 211-230.

Globus, A., and A. B. Scheibel (1967) Synaptic loci in visual cortical neurons of the rabbit: The specific afferent radiation. Exp. Neurol. 18: 116-131.

Gray, E. G. (1959) Axo-somatic and axo-dendritic synapses of the cerebral cortex: An electron microscopic study. J. Anat. 93: 420-433.

Groves, P. M., and C. J. Wilson (1980) Fine structure of rat locus coeruleus. J. Comp. Neurol. 193: 841-852.

Iansek, R., and S. J. Redman (1973) The amplitude, time course and charge of unitary excitatory post-synaptic potentials evoked in spinal motoneurone dendrities. J. Physiol. (Lond.) 234: 665-688.

Jack, J. J. B., and S. J. Redman (1971) An electrical description of the motoneurone, and its application to the analysis of synaptic potentials. J. Physiol. (Lond.) 215: 321-352.

Jack, J. J. B., S. Miller, R. Porter, and S. J. Redman (1971) The time course of minimal excitatory post-synaptic potentials evoked in spinal motoneurones by group Ia afferent fibres. J. Physiol. (Lond.) 215: 353-380.

Jack, J. J. B., D. Noble, and R. W. Tsien (1975) Electric Current Flow in Excitable Cells, pp. 218-223, Oxford University Press, London.

Jan, Y., L. Y. Jan, and S. W. Kuffler (1979) A peptide as a possible transmitter in sympathetic ganglia of the frog. Proc. Natl. Acad. Sci. U. S. A. 76: 1501-1505.

Jones, E. G., and T. P. S. Powell (1969) Morphological variations in the dendritic spines of the neocortex. J. Cell Sci. 5: $509-529$.

Kehoe, J. (1972) The physiological role of three acetylcholine receptors in synaptic transmission in Aplysia. J. Physiol. (Lond.) 225: 147172.

Koch, C., and T. Poggio (1983) Electrical properties of dendritic spines. Trends Neurosci. 6: 80-83.

Libet, B., and T. Tosaka (1969) Slow inhibitory and excitatory postsynaptic responses in single cells of mammalian sympathetic ganglia. J. Neurophysiol. 32: 13-50.
Llinás, R., and D. E. Hillman (1969) Physiological and morphological organization of the cerebellar circuits in various vertebrates. In Neurobiology of Cerebellar Evolution and Development, R. Llinas, ed., pp. 43-73. AMA-ERF Institute for Biomedical Research, Chicago.

Llinás, R., and C. Nicholson (1971) Electrophysiological properties of dendrites and somata in alligator Purkinje cells. J. Neurophysiol. 34: 532-551.

Llinás, R., and M. Sugimori (1980a) Electrophysiological properties of in vitro Purkinje cell somata in mammalian cerebellar slices. J. Physiol. (Lond.) 305: 171-195.

Llinás, R., and M. Sugimori (1980b) Electrophysiological properties of in vitro Purkinje cell dendrites in mammalian cerebellar slices. J. Physiol. (Lond.) 305: 197-213.

MacGregor, R. J. (1968) A model for responses to activation by axodendritic synapses. Biophys. J. 8: 305-318.

Purpura, D. P. (1974) Dendritic spine "dysgenesis" and mental retardation. Science $186: 1126-1128$.

Rall, W. (1959) Branching dendritic trees and motoneuron membrane resistivity. Exp. Neurol. 1: 491-527.

Rall, W. (1960) Membrane potential transients and membrane time constant of motoneurons. Exp. Neurol. 2: 503-532.

Rall, W. (1962) Electrophysiology of a dendritic neuron model. Biophys. J. 2: 145-167.

Rall, W. (1969) Distribution of potential in cylindrical coordinates and time constants for a membrane cylinder. Biophys. J. 9: 1509-1541.

Rall, W. (1974) Dendritic spines, synaptic potency and neuronal plasticity. In Cellular Mechanisms Subserving Changes in Neuronal Activity, C. D. Woody, K. A. Brown, T. J. Crow, and J. D. Knispel, eds., pp. 13-21, Brain Information Service, UCLA, Los Angeles.

Rall, W. (1977) Core conductor theory and cable properties of neurons. In Handbook of Physiology, Sect. 1, Vol. 1: Cellular Biology of Neurons, J. M. Brookhart, V. B. Mountcastle, and E. R. Kandel, eds., pp. 39-97, American Physiological Society, Bethesda, MD.

Rall, W. (1978) Dendritic spines and synaptic potency. In Studies in Neurophysiology, R. Porter, ed., pp. 203-209, Cambridge University Press, New York.

Rall, W., and J. Rinzel (1973) Branch input resistance and steady attenuation for input to one branch of a dendritic neuron model. Biophys. J. 13: 648-688.

Rall, W., R. E. Burke, T. G. Smith, P. G. Nelson, and K. Frank (1967) Dendritic location of synapses and possible mechanisms for the monosynaptic EPSP in motoneurons. J. Neurophysiol. 30: 1169-1193.

Rinzel, J. (1982) Neuronal plasticity (learing). Lect. Math. Life Sci. 15: 7-25.

Rinzel, J., and W. Rall (1974) Transient response in a dendritic neuron model for current injected at one branch. Biophys. J. 14: 759-790.

Scheibel, M. E., and A. B. Scheibel (1968) On the nature of dendritic spines-A report of a workshop. Comm. Behav. Biol. A. 1: 231-265.

Schwartzkroin, P. A., and M. Slawsky (1977) Probable calcium spikes in hippocampal neurons. Brain Res. 135: 157-161.

Shepherd, G. M., and R. K. Brayton (1979) Computer simulation of a dendro-dendritic circuit for self- and lateral-inhibition in the olfactory bulb. Brain Res. 175: 377-382.

Spencer, W. A., and E. R. Kandel (1961) Electrophysiology of hippocampal neurons. IV. Fast prepotentials. J. Neurophysiol. 24: 272-285.

Sugimori, M., R. J. Preston, and S. T. Kitai (1978) Response properties and electrical constants of caudate nucleus neurons in the cat. J. Neurophysiol. 41: 1662-1675.

Takeuchi, A. (1977) Junctional transmission. I. Postsynaptic mechanisms. In Handbook of Physiology Sect. 1, Vol. 1: Cellular Biology of Neurons, J. M. Brookhart, V. B. Mount- 
castle, and E. R. Kandel, eds., pp. 295-327, American Physiological Society, Bethesda, MD.

Tatemichi, R., and E. Ramon-Moliner (1975) Structure of the somatic appendages of neurons of locus coeruleus in cat. Brain Res. 96: 317-322.

Traub, R. D., and R. Llinás (1979) Ilippocampal pyramidal cells: Significance of dendritic ionic conductances for neuronal function and epileptogenesis. J. Neurophysiol. 42: 476496.

Tsukahara, N., F. Murakami, and H. Hultborn (1975) Electrical constants of neurons of the red nucleus. Exp. Brain Res. 23: 49-64.

Valverde, F. (1967) Apical dendritic spines of the visual cortex and light deprivation in the mouse. Exp. Brain Res. 3: 337352 .
Van Harreveld, A., and E. Fifková (1975) Swelling of dendritic spines in fascia dentata after stimulation of the perforant fibers as a mechanism of posttetanic potentiation. Exp. Neurol. 49: 736-749.

Wilson, C. J., and P. M. Groves (1980) Fine structure and synaptic connections of the common spiny neuron of the rat neostriatum: A study employing intracellular injection of horseradish peroxidase. J. Comp. Neurol. 194: 599-616.

Wilson, C. J., P. M. Groves, S. T. Kitai, and J. C. Linder (1983) Three-dimensional structure of dendritic spines in the rat neostriatum. J. Neurosci. 3: 383-398.

Wong, R. K. S., D. A. Prince, and A. I. Basbaum (1979) Intradendritic recordings from hippocampal neurons. Proc. Natl. Acad. Sci. U. S. A. 76: 986-990. 